\title{
On the global regularity of the 2D Oldroyd-B-type model
}

\section{Zhuan $\mathrm{Ye}^{1}$}

Received: 25 April 2018 / Accepted: 1 August 2018 / Published online: 9 August 2018

(c) Fondazione Annali di Matematica Pura ed Applicata and Springer-Verlag GmbH Germany, part of Springer Nature 2018

\section{Abstract}

This paper examines the global regularity problem of the two-dimensional Oldroyd-B-type model. When the initial $L^{2}$-energy is suitably small or the initial stress tensor is nonnegative definite, we show that the corresponding system admits a unique global regular solution.

Keywords Oldroyd-B model · Global regularity

Mathematics Subject Classification 76D03 $\cdot 76 \mathrm{~A} 10 \cdot 35 \mathrm{Q} 35$

\section{Introduction}

The two-dimensional (2D) incompressible Oldroyd-B-type model with diffusive stress can be written as follows:

$$
\left\{\begin{array}{l}
\partial_{t} u+(u \cdot \nabla) u-v \Delta u+\nabla p=\kappa \nabla \cdot \tau, \quad x \in \mathbb{R}^{2}, t>0, \\
\partial_{t} \tau+(u \cdot \nabla) \tau+\zeta \tau-\eta \Delta \tau+Q(\nabla u, \tau)=\gamma \mathcal{D} u, \\
\nabla \cdot u=0, \\
u(x, 0)=u_{0}(x), \tau(x, 0)=\tau_{0}(x),
\end{array}\right.
$$

where the vector $u=\left(u_{1}(x, t), u_{2}(x, t)\right) \in \mathbb{R}^{2}$ is the velocity of the fluid, $p=p(x, t) \in \mathbb{R}$ is the scalar pressure and $\tau=\tau(x, t)$ which is a symmetric tensor is the non-Newtonian part of the stress tensor. The parameters $v, \eta, \zeta, \kappa, \gamma$ are nonnegative constants. Here, $\mathcal{D} u$ is the symmetric part of $\nabla u$, namely $\mathcal{D} u=\frac{1}{2}\left(\nabla u+\nabla u^{\top}\right)$ and $Q$ is a given bilinear form which can be chosen as

$$
Q(\nabla u, \tau)=\Omega \tau-\tau \Omega+b(\mathcal{D} u \tau+\tau \mathcal{D} u),
$$

where $\Omega=\frac{1}{2}\left(\nabla u-\nabla u^{\top}\right)$ is the skew symmetric part of $\nabla u$ and $b \in[-1,1]$ is a parameter. The classical Oldroyd-B model, namely (1.3), with $\mu=0$ originally was introduced by

\section{Zhuan Ye}

yezhuan815@126.com

1 Department of Mathematics and Statistics, Jiangsu Normal University, 101 Shanghai Road, Xuzhou 221116, Jiangsu, People's Republic of China 
Oldroyd [23]. The Oldroyd-B model is one of the basic macroscopic models for viscoelastic flows such as polymer flows. We refer to $[4,11]$ for more details.

Let us first briefly review some existence theories of the Oldroyd-B model from various aspects, and we will not attempt to address exhaustive reference in this paper. Guillopé and Saut in $[12,13]$ examined the classical Oldroyd-B model and proved the existence of local strong solutions in Hilbert space. In the frame of critical Besov spaces, Chemin and Masmoudi [4] first constructed global solutions to the incompressible Oldroyd-B model with small initial data (see Chen and Miao [5] for more general results). Moreover, Chemin and Masmoudi [4] also established some interesting blow-up criteria, which were further improved by Lei et al. [21]. As for the 3D case, Kupferman et al. [18] established a Beale-Kato-Majda-type blowup criterion in terms of $\tau$ in the zero Reynolds number regime. We also point out that Lions and Masmoudi [22] proved the global existence of weak solutions (without uniqueness) of above system (1.1) with $\eta=0$ and $b=0$ (see [3] for more general results). However, the global existence of smooth solutions is open and quite challenging (see [25] for partial answer to this problem). We also mention that global weak of system (1.1) with $\eta=0$ and $b \neq 0$ is still open up to now. As a matter of fact, one notable difference between the Oldroyd-B model and the other fluid equations is that the global-in-time existence of $L^{2}$-estimate has not even been established even in two dimensions. In order to understand this issue, special treatment will needed (see [6,15]). More precisely, the existence and uniqueness of global strong solutions of system (1.1) with $v>0, \mu>0, \gamma=0$ and $b=-1$ was established by Constantin and Kliegel [6] under the assumption of the nonnegativity of the initial stress tensor. By introducing a new quantity $\Gamma=\omega-\mathcal{R} \tau$, where $\omega=\nabla \times u$ and $\mathcal{R}=\frac{\text { curl div }}{\Delta}$, Elgindi and Rousset [8] were able to establish the global regularity with general initial data to system (1.3) in the case $v=0, \mu>0$ and $Q=0$. Moreover, they also obtained the global small initial data in the case of $v=0, \mu>0$ and the general $Q$ given by (1.2). Very recently, there are considerable works devoted to the global existence of smooth solution with small initial data $[7,9,10,14,19,20,26]$.

The first objective of this paper is to consider the following system:

$$
\left\{\begin{array}{l}
\partial_{t} u+(u \cdot \nabla) u+v(-\Delta)^{\alpha} u+\nabla p=\kappa \nabla \cdot \tau, \quad x \in \mathbb{R}^{2}, t>0, \\
\partial_{t} \tau+(u \cdot \nabla) \tau+\zeta \tau+\eta(-\Delta)^{\beta} \tau+Q(\nabla u, \tau)=\gamma \mathcal{D} u, \\
\nabla \cdot u=0, \\
u(x, 0)=u_{0}(x), \tau(x, 0)=\tau_{0}(x),
\end{array}\right.
$$

where $\alpha, \beta \in[0,2]$ and $(-\Delta)^{\gamma}$ denotes the Zygmund operator, defined via the Fourier transform

$$
\widehat{(-\Delta)^{\gamma}} f(\xi)=|\xi|^{2 \gamma} \widehat{f}(\xi), \quad \widehat{f}(\xi)=\int_{\mathbb{R}^{2}} e^{-i x \cdot \xi} f(x) \mathrm{d} x .
$$

We remark the convention that by $\alpha=0$, we mean that there is no dissipation in $(1.3)_{1}$, that is $v=0$, and similarly $\beta=0$ represents that there is no diffusion in $(1.3)_{2}$, that is $\eta=0$. The first two theorems are devoted to establishing the global regularity of the $2 \mathrm{D}$ Oldroyd-B model (1.3) with suitably small initial $L^{2}$-energy. More precisely, the first result can be stated as follows:

Theorem 1.1 Let $\left(u_{0}, \tau_{0}\right) \in H^{s}\left(\mathbb{R}^{2}\right) \times H^{s}\left(\mathbb{R}^{2}\right)$ with $s>2$ and $\nabla \cdot u_{0}=0$. Assume

$$
\gamma\left\|u_{0}\right\|_{L^{2}}^{2}+\kappa\left\|\tau_{0}\right\|_{L^{2}}^{2} \leq \widetilde{C} v \eta \gamma
$$

for some absolute constant $\widetilde{C}$ independent of $\nu, \eta, \kappa$ and $\gamma$. If $\alpha+\beta=2$ with $0<\beta<2$, then $2 D$ Oldroyd-B model (1.3) admits a unique global regular solution $(u, \tau)$ satisfying for 
any given $T>0$,

$$
\begin{aligned}
& u \in L^{\infty}\left([0, T] ; H^{s}\left(\mathbb{R}^{2}\right)\right) \cap L^{2}\left([0, T] ; H^{s+\alpha}\left(\mathbb{R}^{2}\right)\right) ; \\
& \tau \in L^{\infty}\left([0, T] ; H^{s}\left(\mathbb{R}^{2}\right)\right) \cap L^{2}\left([0, T] ; H^{s+\beta}\left(\mathbb{R}^{2}\right)\right) .
\end{aligned}
$$

The second one concerns the borderline case $\alpha=0, \beta=2$.

Theorem 1.2 Consider system (1.3) with $\alpha=0, \beta=2$. Let $\left(u_{0}, \tau_{0}\right) \in H^{s}\left(\mathbb{R}^{2}\right) \times H^{s}\left(\mathbb{R}^{2}\right)$ with $s>2$ and $\nabla \cdot u_{0}=0$. Assume

$$
\gamma\left\|u_{0}\right\|_{L^{2}}^{2}+\kappa\left\|\tau_{0}\right\|_{L^{2}}^{2} \leq \widetilde{C} \zeta \eta \gamma
$$

for some absolute constant $\widetilde{C}$ independent of $\zeta, \eta, \kappa$ and $\gamma$. Then, the $2 D$ Oldroyd-B model (1.3) admits a unique global regular solution $(u, \tau)$ satisfying for any given $T>0$,

$$
u \in L^{\infty}\left([0, T] ; H^{s}\left(\mathbb{R}^{2}\right)\right) ; \quad \tau \in L^{\infty}\left([0, T] ; H^{s}\left(\mathbb{R}^{2}\right)\right) \cap L^{2}\left([0, T] ; H^{s+2}\left(\mathbb{R}^{2}\right)\right) .
$$

Inspired by the work [6], we consider system (1.3) with $b=-1$ and $\gamma=0$,

$$
\left\{\begin{array}{l}
\partial_{t} u+(u \cdot \nabla) u+v(-\Delta)^{\alpha} u+\nabla p=\kappa \nabla \cdot \tau, \quad x \in \mathbb{R}^{2}, t>0, \\
\partial_{t} \tau+(u \cdot \nabla) \tau+\eta(-\Delta)^{\beta} \tau+\zeta \tau=\tau \nabla u+\nabla u^{\top} \tau, \\
\nabla \cdot u=0 \\
u(x, 0)=u_{0}(x), \tau(x, 0)=\tau_{0}(x) .
\end{array}\right.
$$

For system (1.6), our first theorem reads as follows.

Theorem 1.3 Let $s>2$. Assume that $u_{0} \in H^{s}\left(\mathbb{R}^{2}\right)$ is a divergence-free vector and $\tau_{0} \in$ $L^{1}\left(\mathbb{R}^{2}\right) \cap H^{s}\left(\mathbb{R}^{2}\right)$ is a nonnegative definite symmetric matrix. If $\alpha+\beta=2$ with $0 \leq \beta \leq 1$, then $2 D$ Oldroyd-B model (1.6) admits a unique global regular solution $(u, \tau)$ satisfying for any given $T>0$,

$$
\begin{aligned}
& u \in L^{\infty}\left([0, T] ; H^{s}\left(\mathbb{R}^{2}\right)\right) \cap L^{2}\left([0, T] ; H^{s+\alpha}\left(\mathbb{R}^{2}\right)\right) ; \\
& \tau \in L^{\infty}\left([0, T] ; L^{1}\left(\mathbb{R}^{2}\right)\right) \cap L^{\infty}\left([0, T] ; H^{s}\left(\mathbb{R}^{2}\right)\right) \cap L^{2}\left([0, T] ; H^{s+\beta}\left(\mathbb{R}^{2}\right)\right) .
\end{aligned}
$$

Remark 1.4 It is not clear whether Theorem 1.3 holds true for the remainder case, that is, $\alpha+\beta=2$ with $1<\beta<2$. The key reason is that the proof of Theorem 1.3 relies heavily on Lemma 4.1. However, the validity of Lemma 4.1 requires the restriction $0 \leq \beta \leq 1$.

The second one is to consider system (1.6) with the borderline case $\alpha=2, \beta=0$. More precisely, we have the following logarithmically improved result.

Theorem 1.5 Consider the following system

$$
\left\{\begin{array}{l}
\partial_{t} u+(u \cdot \nabla) u+v \mathcal{L}^{2} u+\nabla p=\kappa \nabla \cdot \tau \\
\partial_{t} \tau+(u \cdot \nabla) \tau+\zeta \tau=\tau \nabla u+\nabla u^{\top} \tau \\
\nabla \cdot u=0 \\
u(x, 0)=u_{0}(x), \tau(x, 0)=\tau_{0}(x)
\end{array}\right.
$$

where the operator $\mathcal{L}$ is defined by

$$
\widehat{\mathcal{L} u}(\xi)=\frac{|\xi|^{2}}{g(|\xi|)} \widehat{u}(\xi)
$$


for some radially symmetric, non-decreasing function $g(\tau) \geq 1$ defined on $\tau \geq 0$. Assume that $u_{0} \in H^{s}\left(\mathbb{R}^{2}\right)$ with $s>2$ is a divergence-free vector and $\tau_{0} \in L^{1}\left(\mathbb{R}^{2}\right) \cap H^{s}\left(\mathbb{R}^{2}\right)$ is a nonnegative definite symmetric matrix. If $g$ satisfies the following growth condition

$$
\int_{e}^{\infty} \frac{d \tau}{\tau \sqrt{\ln \tau} g(\tau)}=\infty,
$$

then system (1.7) admits a unique global regular solution $(u, \tau)$ such that for any given $T>0$,

$$
(u, \tau) \in L^{\infty}\left([0, T] ; H^{s}\left(\mathbb{R}^{n}\right)\right), \quad \mathcal{L} u \in L^{2}\left([0, T] ; H^{s}\left(\mathbb{R}^{n}\right)\right) .
$$

In this paper, all constants will be denoted by $C$ that is a generic constant depending only on the quantities specified in the context. We shall write $C\left(\gamma_{1}, \gamma_{2}, \ldots, \gamma_{k}\right)$ as the constant $C$ depends on the quantities $\gamma_{1}, \gamma_{2}, \ldots, \gamma_{k}$. We point out that for two matrices $\mathbb{A}$ and $\mathbb{B}$ of size $n \times n$, we denote $\mathbb{A}: \mathbb{B}=\sum_{i, j=1}^{n} a_{i j} b_{i j}$, where $a_{i j}$ and $b_{i j}$ are the components of matrices $\mathbb{A}$ and $\mathbb{B}$, respectively. To simplify the presentation, we denote $\Lambda:=(-\Delta)^{\frac{1}{2}}$ throughout this paper.

The paper is organized as follows. In Sect. 2, we give the proof of Theorem 1.1. An overview of the proof of Theorem 1.2 is given in Sect. 3. Sections 4 and 5 are devoted to the proof of Theorems 1.3 and 1.5, respectively. For the reader's convenience, the Besov spaces and the Bernstein inequality are collected in Appendix.

\section{The proof of Theorem 1.1}

This section is devoted to the proof of Theorem 1.1. The existence and uniqueness of local smooth solutions in $H^{s}\left(\mathbb{R}^{2}\right)$ with $s>2$ can be obtained via standard approach; thus, it is sufficient to establish a priori estimates for $(u, \tau)$ at the interval $[0, T]$ for any given $T>0$. We start with the following global $L^{2}$-estimate for system (1.3).

Lemma 2.1 Assume that $\left(u_{0}, \tau_{0}\right)$ satisfies the assumptions stated in Theorem 1.1. Let $(u, \tau)$ be the corresponding smooth solution of (1.3). If $\alpha+\beta=2$ with $0<\beta<2$, then $(u, \tau)$ obeys the global bound

$$
\|u(t)\|_{L^{2}}^{2}+\|\tau(t)\|_{L^{2}}^{2}+\int_{0}^{t}\left(\left\|\Lambda^{\alpha} u(s)\right\|_{L^{2}}^{2}+\left\|\Lambda^{\beta} \tau(s)\right\|_{L^{2}}^{2}\right) d s \leq C\left(u_{0}, \tau_{0}\right) .
$$

Proof of Lemma 2.1 Multiplying (1.3) 1 by $\gamma u$ and (1.3) 2 by $\kappa \tau$ and integrating over $\mathbb{R}^{2}$, we obtain by adding them up

$$
\begin{aligned}
& \frac{1}{2} \frac{\mathrm{d}}{\mathrm{d} t}\left(\gamma\|u(t)\|_{L^{2}}^{2}+\kappa\|\tau(t)\|_{L^{2}}^{2}\right)+v \gamma\left\|\Lambda^{\alpha} u\right\|_{L^{2}}^{2}+\kappa \eta\left\|\Lambda^{\beta} \tau\right\|_{L^{2}}^{2}+\kappa \zeta\|\tau\|_{L^{2}}^{2} \\
& \quad=\kappa \int_{\mathbb{R}^{2}} Q(\nabla u, \tau): \tau \mathrm{d} x
\end{aligned}
$$

where we have used the cancelation

$$
\int_{\mathbb{R}^{2}}(\nabla \cdot \tau) \cdot u \mathrm{~d} x+\int_{\mathbb{R}^{2}} \mathcal{D} u: \tau \mathrm{d} x=0 .
$$


On the one hand, for the case $0<\alpha \leq 1$ or $1 \leq \beta<2$, we have

$$
\begin{aligned}
\kappa \int_{\mathbb{R}^{2}} Q(\nabla u, \tau): \tau \mathrm{d} x & \leq C \kappa\left\|\Lambda^{\alpha} u\right\|_{L^{2}}\left\|\Lambda^{1-\alpha}(\tau \tau)\right\|_{L^{2}} \\
& \leq C \kappa\left\|\Lambda^{\alpha} u\right\|_{L^{2}}\|\tau\|_{L^{6}}\left\|\Lambda^{1-\alpha} \tau\right\|_{L^{3}} \\
& \leq C \kappa\left\|\Lambda^{\alpha} u\right\|_{L^{2}}\left(\|\tau\|_{L^{2}}^{1-\frac{2}{3 \beta}}\left\|\Lambda^{\beta} \tau\right\|_{L^{2}}^{\frac{2}{3 \beta}}\right)\left(\|\tau\|_{L^{2}}^{\frac{2}{3 \beta}}\left\|\Lambda^{\beta} \tau\right\|_{L^{2}}^{1-\frac{2}{3 \beta}}\right) \\
& =C \kappa\left\|\Lambda^{\alpha} u\right\|_{L^{2}}\|\tau\|_{L^{2}}\left\|\Lambda^{\beta} \tau\right\|_{L^{2}} \\
& \leq \frac{v \gamma}{2}\left\|\Lambda^{\alpha} u\right\|_{L^{2}}^{2}+C \frac{\kappa^{2}}{v \gamma}\|\tau\|_{L^{2}}^{2}\left\|\Lambda^{\beta} \tau\right\|_{L^{2}}^{2} \\
& \leq \frac{v \gamma}{2}\left\|\Lambda^{\alpha} u\right\|_{L^{2}}^{2}+C \frac{\kappa}{v \gamma}\left(\gamma\|u\|_{L^{2}}^{2}+\kappa\|\tau\|_{L^{2}}^{2}\right)\left\|\Lambda^{\beta} \tau\right\|_{L^{2}}^{2},
\end{aligned}
$$

where we have applied the following product estimate (see [16])

$$
\left\|\Lambda^{s}(f g)\right\|_{L^{p}} \leq C\left(\|f\|_{L^{p_{1}}}\left\|\Lambda^{s} g\right\|_{L^{p_{2}}}+\left\|\Lambda^{s} f\right\|_{L^{p_{3}}}\|g\|_{L^{p_{4}}}\right),
$$

with $s>0, p_{1}, p_{4} \in(1, \infty], p_{2}, p_{3} \in(1, \infty)$ satisfying $\frac{1}{p}=\frac{1}{p_{1}}+\frac{1}{p_{2}}=\frac{1}{p_{3}}+\frac{1}{p_{4}}$. On the other hand, for the case $1<\alpha<2$ or $0<\beta<1$, we have

$$
\begin{aligned}
\kappa \int_{\mathbb{R}^{2}} Q(\nabla u, \tau): \tau \mathrm{d} x & \leq C \kappa\|\nabla u\|_{L^{\frac{2}{2-\alpha}}}\|\tau \tau\|_{L^{\frac{2}{\alpha}}} \\
& \leq C \kappa\left\|\Lambda^{\alpha} u\right\|_{L^{2}}\|\tau\|_{L^{\frac{4}{\alpha}}}^{2} \\
& \leq C \kappa\left\|\Lambda^{\alpha} u\right\|_{L^{2}}\|\tau\|_{L^{2}}\left\|\Lambda^{\beta} \tau\right\|_{L^{2}} \\
& \leq \frac{v \gamma}{2}\left\|\Lambda^{\alpha} u\right\|_{L^{2}}^{2}+C \frac{\kappa}{v \gamma}\left(\gamma\|u\|_{L^{2}}^{2}+\kappa\|\tau\|_{L^{2}}^{2}\right)\left\|\Lambda^{\beta} \tau\right\|_{L^{2}}^{2} .
\end{aligned}
$$

Inserting the above estimates into (2.2) yields

$$
\begin{aligned}
\frac{\mathrm{d}}{\mathrm{d} t}\left(\gamma\|u(t)\|_{L^{2}}^{2}+\kappa\|\tau(t)\|_{L^{2}}^{2}\right) & +v \gamma\left\|\Lambda^{\alpha} u\right\|_{L^{2}}^{2}+\kappa \eta\left\|\Lambda^{\beta} \tau\right\|_{L^{2}}^{2} \\
& \leq C \frac{\kappa}{v \gamma}\left(\gamma\|u\|_{L^{2}}^{2}+\kappa\|\tau\|_{L^{2}}^{2}\right)\left\|\Lambda^{\beta} \tau\right\|_{L^{2}}^{2} .
\end{aligned}
$$

Under the assumption (1.4), we claim that if

$$
\gamma\left\|u_{0}\right\|_{L^{2}}^{2}+\kappa\left\|\tau_{0}\right\|_{L^{2}}^{2} \leq \frac{v \eta \gamma}{4 C}
$$

then the following holds

$$
\gamma\|u(t)\|_{L^{2}}^{2}+\kappa\|\tau(t)\|_{L^{2}}^{2}<\frac{v \eta \gamma}{2 C}, \forall t>0
$$

Now suppose that (2.5) is not true and $\widetilde{T}$ is the first time such that (2.5) is violated. Namely, we have

$$
\gamma\|u(\widetilde{T})\|_{L^{2}}^{2}+\kappa\|\tau(\widetilde{T})\|_{L^{2}}^{2}=\frac{v \eta \gamma}{2 C}
$$

moreover,

$$
\gamma\|u(t)\|_{L^{2}}^{2}+\kappa\|\tau(t)\|_{L^{2}}^{2}<\frac{\nu \eta \gamma}{2 C}, \quad \forall 0 \leq t<\widetilde{T} .
$$


In view of (2.4), it follows that for any $0 \leq t \leq \widetilde{T}$

$$
\frac{\mathrm{d}}{\mathrm{d} t}\left(\gamma\|u(t)\|_{L^{2}}^{2}+\kappa\|\tau(t)\|_{L^{2}}^{2}\right)+v \gamma\left\|\Lambda^{\alpha} u\right\|_{L^{2}}^{2}+\kappa \eta\left\|\Lambda^{\beta} \tau\right\|_{L^{2}}^{2} \leq \frac{\kappa \eta}{2}\left\|\Lambda^{\beta} \tau\right\|_{L^{2}}^{2},
$$

or

$$
\frac{\mathrm{d}}{\mathrm{d} t}\left(\gamma\|u(t)\|_{L^{2}}^{2}+\kappa\|\tau(t)\|_{L^{2}}^{2}\right)+v \gamma\left\|\Lambda^{\alpha} u\right\|_{L^{2}}^{2}+\frac{\kappa \eta}{2}\left\|\Lambda^{\beta} \tau\right\|_{L^{2}}^{2} \leq 0 .
$$

Therefore, we have for any $0 \leq t \leq \widetilde{T}$

$$
\gamma\|u(t)\|_{L^{2}}^{2}+\kappa\|\tau(t)\|_{L^{2}}^{2}+\int_{0}^{t}\left(v \gamma\left\|\Lambda^{\alpha} u\right\|_{L^{2}}^{2}+\frac{\kappa \eta}{2}\left\|\Lambda^{\beta} \tau\right\|_{L^{2}}^{2}\right)(s) \mathrm{d} s \leq \frac{v \eta \gamma}{4 C} .
$$

In particular, it gives

$$
\gamma\|u(\widetilde{T})\|_{L^{2}}^{2}+\kappa\|\tau(\widetilde{T})\|_{L^{2}}^{2}<\frac{\nu \eta \gamma}{4 C},
$$

which contradicts with (2.6). This contradiction implies that (2.5) holds true. Now coming back to (2.4), we have

$$
\frac{\mathrm{d}}{\mathrm{d} t}\left(\gamma\|u(t)\|_{L^{2}}^{2}+\kappa\|\tau(t)\|_{L^{2}}^{2}\right)+v \gamma\left\|\Lambda^{\alpha} u\right\|_{L^{2}}^{2}+\frac{\kappa \eta}{2}\left\|\Lambda^{\beta} \tau\right\|_{L^{2}}^{2} \leq 0 .
$$

Integrating the above inequality from 0 to $t$, we obtain the desired result (2.1). This ends the proof of the lemma.

We will prove the following estimate, which allows us to establish the higher regularity for $u$ and $\tau$.

Lemma 2.2 Assume that $\left(u_{0}, \tau_{0}\right)$ satisfies the assumptions stated in Theorem 1.1. Let $(u, \tau)$ be the corresponding smooth solution of (1.3). If $\alpha+\beta=2$ with $0<\beta<2$, then $(u, \tau)$ obeys the global bound

$$
\|\tau(t)\|_{L^{p}}+\int_{0}^{t}\|\nabla u(s)\|_{L^{q}}^{2} d s \leq C\left(p, q, t, u_{0}, \tau_{0}\right)
$$

for any $p, q \in[2, \infty)$. As a matter of fact, for the case $\alpha+\beta=2$ with $0<\beta<2$ and $\beta \neq 1$, (2.7) can be at least improved as

$$
\|\tau(t)\|_{L^{\infty}}+\int_{0}^{t}\|\omega(s)\|_{L^{\infty}}^{2} d s \leq C\left(t, u_{0}, \tau_{0}\right),
$$

where $\omega$ is the vorticity of the velocity $u$, namely, $\omega=\partial_{x_{1}} u_{2}-\partial_{x_{2}} u_{1}$.

Proof of Lemma 2.2 Testing $(1.3)_{1}$ by $-\Delta u$, using the divergence-free condition and noticing the following identity (see [24, (3.3)] for details)

$$
\int_{\mathbb{R}^{2}}(u \cdot \nabla u) \cdot \Delta u \mathrm{~d} x=0,
$$

we obtain

$$
\begin{aligned}
\frac{1}{2} \frac{\mathrm{d}}{\mathrm{d} t}\|\nabla u(t)\|_{L^{2}}^{2}+v\left\|\Lambda^{\alpha} \nabla u\right\|_{L^{2}}^{2} & =-\kappa \int_{\mathbb{R}^{2}} \nabla \cdot \tau \cdot \Delta u \mathrm{~d} x \\
& \leq C\left\|\Lambda^{\beta} \tau\right\|_{L^{2}}\left\|\Lambda^{\alpha} \nabla u\right\|_{L^{2}} \\
& \leq \frac{v}{2}\left\|\Lambda^{\alpha} \nabla u\right\|_{L^{2}}^{2}+C\left\|\Lambda^{\beta} \tau\right\|_{L^{2}}^{2} .
\end{aligned}
$$


Thanks to (2.1), we get

$$
\|\nabla u(t)\|_{L^{2}}^{2}+\int_{0}^{t}\left\|\Lambda^{\alpha} \nabla u(s)\right\|_{L^{2}}^{2} \mathrm{~d} s \leq C\left(u_{0}, \tau_{0}\right)
$$

We first consider the case $\alpha \in[1,2)$. It directly leads to

$$
\int_{0}^{t}\|\nabla u(s)\|_{L^{q}}^{2} \mathrm{~d} s \leq C\left(q, t, u_{0}, \tau_{0}\right)
$$

by using

$$
\|\nabla u\|_{L^{q}} \leq C\|\nabla u\|_{L^{2}}^{1-\frac{q-2}{\alpha q}}\left\|\Lambda^{\alpha} \nabla u\right\|_{L^{2}}^{\frac{q-2}{\alpha q}}, \quad \forall q \in[2, \infty)
$$

We point out that when $\alpha>1$, the constant $C\left(q, t, u_{0}, \tau_{0}\right)$ of (2.8) can be replaced by $C\left(t, u_{0}, \tau_{0}\right)$. Now multiplying the equation $(1.3)_{2}$ by $|\tau|^{p-2} \tau$, integrating over $\mathbb{R}^{2}$ and using the divergence-free condition, we obtain

$$
\begin{aligned}
& \frac{1}{p} \frac{\mathrm{d}}{\mathrm{d} t}\|\tau(t)\|_{L^{p}}^{p}+\zeta\|\tau\|_{L^{p}}^{p}+\eta \int_{\mathbb{R}^{2}}\left(\Lambda^{2 \beta} \tau\right): \tau|\tau|^{p-2} \mathrm{~d} x \\
& \quad=\gamma \int_{\mathbb{R}^{2}} \mathcal{D} u: \tau|\tau|^{p-2} \mathrm{~d} x-\int_{\mathbb{R}^{2}} Q(\nabla u, \tau): \tau|\tau|^{p-2} \mathrm{~d} x .
\end{aligned}
$$

If $\alpha=\beta=1$, then we may deduce from (2.11) by integrating by parts

$$
\begin{aligned}
\frac{1}{p} & \frac{\mathrm{d}}{\mathrm{d} t}\|\tau(t)\|_{L^{p}}^{p}+\eta \int_{\mathbb{R}^{2}}|\nabla \tau|^{2}|\tau|^{p-2} \mathrm{~d} x \\
& \leq C\|\nabla u\|_{L^{p}}\|\tau\|_{L^{p}}^{p-1}+\left.C\left|\int_{\mathbb{R}^{2}} u \nabla \tau\right| \tau\right|^{p-1} \mathrm{~d} x \mid \\
& \leq \frac{\eta}{2} \int_{\mathbb{R}^{2}}|\nabla \tau|^{2}|\tau|^{p-2} \mathrm{~d} x+C\|\nabla u\|_{L^{p}}\|\tau\|_{L^{p}}^{p-1}+C \int_{\mathbb{R}^{2}}|u|^{2}|\tau|^{p} \mathrm{~d} x \\
& \leq \frac{\eta}{2} \int_{\mathbb{R}^{2}}|\nabla \tau|^{2}|\tau|^{p-2} \mathrm{~d} x+C\|\nabla u\|_{L^{p}}\|\tau\|_{L^{p}}^{p-1}+C\|u\|_{L^{\infty}}^{2}\|\tau\|_{L^{p}}^{p} \\
& \leq \frac{\eta}{2} \int_{\mathbb{R}^{2}}|\nabla \tau|^{2}|\tau|^{p-2} \mathrm{~d} x+C\left(1+\|u\|_{L^{2}}^{2}+\|\nabla u\|_{L^{p}}^{2}\right)\left(1+\|\tau\|_{L^{p}}^{p}\right) .
\end{aligned}
$$

This gives

$$
\frac{\mathrm{d}}{\mathrm{d} t}\|\tau(t)\|_{L^{p}}^{p} \leq C\left(1+\|u\|_{L^{2}}^{2}+\|\nabla u\|_{L^{p}}^{2}\right)\left(1+\|\tau\|_{L^{p}}^{p}\right) .
$$

Gronwall inequality ensures

$$
\|\tau(t)\|_{L^{p}} \leq C\left(p, t, u_{0}, \tau_{0}\right)
$$

For the case $\alpha \in(1,2)$ or $\beta \in(0,1)$, we first have the lower bound

$$
\int_{\mathbb{R}^{2}}\left(\Lambda^{2 \beta} \tau\right): \tau|\tau|^{p-2} \mathrm{~d} x \geq c\left\|\Lambda^{\beta}\left(|\tau|^{\frac{p}{2}}\right)\right\|_{L^{2}}^{2} \geq c\|\tau\|_{L^{\frac{p}{1-\beta}}}^{p} .
$$


Consequently, one gets

$$
\begin{aligned}
\frac{1}{p} \frac{\mathrm{d}}{\mathrm{d} t}\|\tau(t)\|_{L^{p}}^{p}+\eta c\|\tau\|_{L^{\frac{p}{1-\beta}}}^{p} & \leq \gamma \int_{\mathbb{R}^{2}} \mathcal{D} u: \tau|\tau|^{p-2} \mathrm{~d} x-\int_{\mathbb{R}^{2}} Q(\nabla u, \tau): \tau|\tau|^{p-2} \mathrm{~d} x \\
& \leq C\|\nabla u\|_{L^{p}}\|\tau\|_{L^{p}}^{p-1}+C\|\nabla u\|_{L^{\frac{2}{\beta}}\|\tau\|_{L^{\frac{2 p}{2-\beta}}}^{p}} \\
\leq & C\|\nabla u\|_{L^{p}}\|\tau\|_{L^{p}}^{p-1}+C\|\nabla u\|_{L^{\frac{2}{\beta}}\|\tau\|_{L^{p}}^{\frac{p}{2}}\|\tau\|_{L^{\frac{p}{1}-\beta}}^{\frac{p}{2}}} \\
& \leq \frac{\eta c}{2}\|\tau\|_{L^{\frac{p}{1-\beta}}}^{p}+C\|\nabla u\|_{L^{p}}\|\tau\|_{L^{p}}^{p-1}+C\|\nabla u\|_{L^{\frac{2}{\beta}}}^{2}\|\tau\|_{L^{p}}^{p} \\
\leq & \frac{\eta c}{2}\|\tau\|_{L^{\frac{p}{1-\beta}}}^{p}+C\left(\|\nabla u\|_{L^{p}}+\|\nabla u\|_{L^{\frac{2}{\beta}}}^{2}\right)\left(1+\|\tau\|_{L^{p}}^{p}\right),
\end{aligned}
$$

which implies

$$
\frac{\mathrm{d}}{\mathrm{d} t}\|\tau(t)\|_{L^{p}}^{p} \leq C\left(\|\nabla u\|_{L^{p}}+\|\nabla u\|_{L^{\frac{2}{\beta}}}^{2}\right)\left(1+\|\tau\|_{L^{p}}^{p}\right) .
$$

Noticing (2.10) and applying the Gronwall inequality to (2.13), we conclude

$$
\|\tau(t)\|_{L^{p}} \leq C\left(p, t, u_{0}, \tau_{0}\right) .
$$

Therefore, the desired estimate (2.7) is proved for the case $\alpha \in[1,2)$ by combining (2.10) and (2.14).

Now we focus on the remainder case, that is $\alpha \in(0,1)$ or $\beta \in(1,2)$. This case is much more involved due to the absence of (2.10). To overcome this difficulty, we make our efforts to obtain the higher regularity for $\tau$. To this end, applying $\Lambda^{2}$ to $(1.3)_{2}$ and multiplying it by $\Lambda^{2} \tau$, one deduces

$$
\frac{1}{2} \frac{\mathrm{d}}{\mathrm{d} t}\left\|\Lambda^{2} \tau(t)\right\|_{L^{2}}^{2}+\eta\left\|\Lambda^{2+\beta} \tau\right\|_{L^{2}}^{2}+\zeta\left\|\Lambda^{2} \tau\right\|_{L^{2}}^{2}=J_{1}+J_{2}+J_{3},
$$

where

$$
\begin{aligned}
& J_{1}=-\int_{\mathbb{R}^{2}} \Lambda^{2} Q(\nabla u, \tau): \Lambda^{2} \tau \mathrm{d} x, \quad J_{2}=-\int_{\mathbb{R}^{2}} \Lambda^{2}(u \cdot \nabla \tau): \Lambda^{2} \tau \mathrm{d} x, \\
& J_{3}=\gamma \int_{\mathbb{R}^{2}} \Lambda^{2} \mathcal{D} u: \Lambda^{2} \tau \mathrm{d} x .
\end{aligned}
$$

By the Young's inequality and (2.3), it gives

$$
\begin{aligned}
J_{1} & \leq C\left\|\Lambda^{2-\beta} Q(\nabla u, \tau)\right\|_{L^{2}}\left\|\Lambda^{2+\beta} \tau\right\|_{L^{2}} \\
& \leq C\left(\|\tau\|_{L^{\infty}}\left\|\Lambda^{3-\beta} u\right\|_{L^{2}}+\left\|\Lambda^{2-\beta} \tau\right\|_{L^{\frac{2}{\alpha}}}\|\nabla u\|_{L^{\frac{2}{1-\alpha}}}\right)\left\|\Lambda^{2+\beta} \tau\right\|_{L^{2}} \\
& \leq C\left(\|\tau\|_{L^{\infty}}\left\|\Lambda^{\alpha} \nabla u\right\|_{L^{2}}+\|\nabla \tau\|_{L^{2}}\left\|\Lambda^{\alpha} \nabla u\right\|_{L^{2}}\right)\left\|\Lambda^{2+\beta} \tau\right\|_{L^{2}} \\
& \leq \frac{\eta}{8}\left\|\Lambda^{2+\beta} \tau\right\|_{L^{2}}^{2}+C\|u\|_{H^{1+\alpha}}^{2}\left\|\Lambda^{2} \tau\right\|_{L^{2}}^{2}+C\|u\|_{H^{1+\alpha}}^{2}\|\tau\|_{L^{2}}^{2} .
\end{aligned}
$$

Similarly, noticing $\nabla \cdot u=0$, we also obtain

$$
\begin{aligned}
J_{2} & \leq C\left\|\Lambda^{3-\beta}(\tau u)\right\|_{L^{2}}\left\|\Lambda^{2+\beta} \tau\right\|_{L^{2}} \\
& \leq C\left(\|\tau\|_{L^{\infty}}\left\|\Lambda^{3-\beta} u\right\|_{L^{2}}+\|u\|_{L^{\infty}}\left\|\Lambda^{3-\beta} \tau\right\|_{L^{2}}\right)\left\|\Lambda^{2+\beta} \tau\right\|_{L^{2}} \\
& \leq C\|\tau\|_{H^{2}}\|u\|_{H^{1+\alpha}}\left\|\Lambda^{2+\beta} \tau\right\|_{L^{2}} \\
& \leq \frac{\eta}{8}\left\|\Lambda^{2+\beta} \tau\right\|_{L^{2}}^{2}+C\|u\|_{H^{1+\alpha}}^{2}\left\|\Lambda^{2} \tau\right\|_{L^{2}}^{2}+C\|u\|_{H^{1+\alpha}}^{2}\|\tau\|_{L^{2}}^{2} .
\end{aligned}
$$


In view of the Young's inequality, one has

$$
J_{3} \leq C\left\|\Lambda^{2+\beta} \tau\right\|_{L^{2}}\left\|\Lambda^{\alpha} \nabla u\right\|_{L^{2}} \leq \frac{\eta}{8}\left\|\Lambda^{2+\beta} \tau\right\|_{L^{2}}^{2}+C\|u\|_{H^{1+\alpha}}^{2} .
$$

Inserting the above estimates into (2.15) yields

$$
\frac{\mathrm{d}}{\mathrm{d} t}\left\|\Lambda^{2} \tau(t)\right\|_{L^{2}}^{2}+\left\|\Lambda^{2+\beta} \tau\right\|_{L^{2}}^{2} \leq C\|u\|_{H^{1+\alpha}}^{2}\left\|\Lambda^{2} \tau\right\|_{L^{2}}^{2}+C\left(1+\|\tau\|_{L^{2}}^{2}\right)\|u\|_{H^{1+\alpha}}^{2} .
$$

Thanks to the Gronwall inequality and (2.1) as well as (2.9), it follows that

$$
\left\|\Lambda^{2} \tau(t)\right\|_{L^{2}}^{2}+\int_{0}^{t}\left\|\Lambda^{2+\beta} \tau(s)\right\|_{L^{2}}^{2} \mathrm{~d} s \leq C\left(t, u_{0}, \tau_{0}\right) .
$$

The vorticity $\omega$ of $(1.3)_{1}$ reads

$$
\partial_{t} \omega+(u \cdot \nabla) \omega+v(-\Delta)^{\alpha} \omega=\kappa \nabla \times \nabla \cdot \tau .
$$

Multiplying (2.17) by $|\omega|^{q-2} \omega$, integrating over $\mathbb{R}^{2}$ and using the following fact

$$
v \int_{\mathbb{R}^{2}}(-\Delta)^{\alpha} \omega\left(|\omega|^{q-2} \omega\right) \mathrm{d} x \geq 0
$$

one obtains for any $q \geq 2$

$$
\begin{aligned}
\frac{1}{q} \frac{\mathrm{d}}{\mathrm{d} t}\|\omega(t)\|_{L^{q}}^{q} & \leq \kappa\left|\int_{\mathbb{R}^{2}} \nabla \times \nabla \cdot \tau\left(|\omega|^{q-2} \omega\right) \mathrm{d} x\right| \\
& \leq C\|\nabla \times \nabla \cdot \tau\|_{L^{q}\|\omega(t)\|_{L^{q}}^{q-1}} \\
& \leq C\left\|\Lambda^{2} \tau\right\|_{H^{\beta}}\|\omega(t)\|_{L^{q}}^{q-1} .
\end{aligned}
$$

This implies

$$
\frac{\mathrm{d}}{\mathrm{d} t}\|\omega(t)\|_{L^{q}} \leq C\left\|\Lambda^{2} \tau\right\|_{H^{\beta}} .
$$

Using (2.16) and integrating (2.18) in time yield

$$
\|\omega(t)\|_{L^{q}} \leq C\left(t, u_{0}, \tau_{0}\right) .
$$

We thus obtain

$$
\|\nabla u(t)\|_{L^{q}} \leq C(q)\|\omega(t)\|_{L^{q}} \leq C\left(t, q, u_{0}, \tau_{0}\right) .
$$

Finally, for $\alpha+\beta=2$ with $0<\beta<2$, the desired estimate (2.7) follows directly. This ends the proof of Lemma 2.2.

Now we are ready to prove the global $H^{s}$-bound with the obtained estimates at our disposal.

Proof Applying the operator $\Lambda^{s}$ with $s>2$ to system (1.3), taking the $L^{2}$ inner product with $\Lambda^{s} u$ and $\Lambda^{s} \tau$, respectively, and adding them up, we can get

$$
\frac{1}{2} \frac{\mathrm{d}}{\mathrm{d} t}\left(\left\|\Lambda^{s} u(t)\right\|_{L^{2}}^{2}+\left\|\Lambda^{s} \tau(t)\right\|_{L^{2}}^{2}\right)+v\left\|\Lambda^{s+\alpha} u\right\|_{L^{2}}^{2}+\eta\left\|\Lambda^{s+\beta} \tau\right\|_{L^{2}}^{2} \leq N_{1}+N_{2}+N_{3}+N_{4},
$$

where

$$
\begin{aligned}
& N_{1}:=\int_{\mathbb{R}^{2}}\left[\Lambda^{s}, u \cdot \nabla\right] u \cdot \Lambda^{s} u \mathrm{~d} x, \quad N_{2}:=\int_{\mathbb{R}^{2}}\left[\Lambda^{s}, u \cdot \nabla\right] \tau: \Lambda^{s} \tau \mathrm{d} x \\
& N_{3}:=-\int_{\mathbb{R}^{2}} \Lambda^{s} Q(\nabla u, \tau): \Lambda^{s} \tau \mathrm{d} x, \quad N_{4}:=\kappa \int_{\mathbb{R}^{2}} \Lambda^{s} \nabla \cdot \tau \cdot \Lambda^{s} u \mathrm{~d} x+\gamma \int_{\mathbb{R}^{2}} \Lambda^{s} \mathcal{D} u: \Lambda^{s} \tau \mathrm{d} x .
\end{aligned}
$$


Recall the following commutator estimate (see [16])

$$
\left\|\left[\Lambda^{s}, f\right] g\right\|_{L^{p}} \leq C\left(\|\nabla f\|_{L^{p_{1}}}\left\|\Lambda^{s-1} g\right\|_{L^{p_{2}}}+\left\|\Lambda^{s} f\right\|_{L^{p_{3}}}\|g\|_{L^{p_{4}}}\right),
$$

with $s>0, p_{1}, p_{4} \in(1, \infty], p_{2}, p_{3} \in(1, \infty)$ satisfying $\frac{1}{p}=\frac{1}{p_{1}}+\frac{1}{p_{2}}=\frac{1}{p_{3}}+\frac{1}{p_{4}}$. We remark that the following variant version of the above commutator estimate also holds true

$$
\left\|\left[\Lambda^{s-1} \partial_{i}, f\right] g\right\|_{L^{r}} \leq C\left(\|\nabla f\|_{L^{p_{1}}}\left\|\Lambda^{s-1} g\right\|_{L^{p_{2}}}+\left\|\Lambda^{s} f\right\|_{L^{p_{3}}}\|g\|_{L^{p_{4}}}\right) .
$$

As a result, we can show

$$
\begin{aligned}
N_{1} & \leq C\left\|\left[\Lambda^{s}, u \cdot \nabla\right] u\right\|_{L^{\frac{2 q_{0}}{q_{0}+1}}}\left\|\Lambda^{s} u\right\|_{L^{\frac{2 q_{0}}{q_{0}}}} \\
& \leq C\|\nabla u\|_{L^{q 0}}\left\|\Lambda^{s} u\right\|_{L^{2}}^{2} \\
& \leq C\|\nabla u\|_{L^{q_{0}}}\left\|\Lambda^{s} u\right\|_{L^{2}}^{2\left(1-\frac{1}{\alpha q_{0}}\right)}\left\|\Lambda^{s+\alpha} u\right\|_{L^{2}}^{\frac{2}{\alpha q_{0}}} \\
& \leq \frac{v}{8}\left\|\Lambda^{s+\alpha} u\right\|_{L^{2}}^{2}+C\|\nabla u\|_{L^{q_{0}}}^{\frac{\alpha q_{0}}{\alpha q_{0}}}\left\|\Lambda^{s} u\right\|_{L^{2}}^{2} \\
& \leq \frac{v}{8}\left\|\Lambda^{s+\alpha} u\right\|_{L^{2}}^{2}+C\left(1+\|\nabla u\|_{L^{q_{0}}}^{2}\right)\left\|\Lambda^{s} u\right\|_{L^{2}}^{2},
\end{aligned}
$$

where $q_{0} \geq \max \left\{2, \frac{2}{\alpha}\right\}$. For the case $\beta \in(1,2)$, we have

$$
\begin{aligned}
& N_{2}=\int_{\mathbb{R}^{2}}\left[\Lambda^{s}, u \cdot \nabla\right] \tau: \Lambda^{s} \tau \mathrm{d} x \\
& \leq C\left\|\left[\Lambda^{s}, u \cdot \nabla\right] \tau\right\|_{L^{2}}\left\|\Lambda^{s} \tau\right\|_{L^{2}} \\
& \leq C\left(\|\nabla u\|_{L^{q_{0}}}\left\|\Lambda^{s} \tau\right\|_{L^{\frac{2 q_{0}}{q_{0}-2}}}+\|\nabla \tau\|_{L^{\infty}}\left\|\Lambda^{s} u\right\|_{L^{2}}\right)\left\|\Lambda^{s} \tau\right\|_{L^{2}} \\
& \leq C\left(\|\nabla u\|_{L^{q_{0}}}\left\|\Lambda^{s} \tau\right\|_{L^{2}}^{1-\frac{2}{\beta q_{0}}}\left\|\Lambda^{s+\beta} \tau\right\|_{L^{2}}^{\frac{2}{\beta q_{0}}}+\|\tau\|_{H^{2+\beta}}\left\|\Lambda^{s} u\right\|_{L^{2}}\right)\left\|\Lambda^{s} \tau\right\|_{L^{2}} \\
& \leq \frac{\eta}{8}\left\|\Lambda^{s+\beta} \tau\right\|_{L^{2}}^{2}+C\left(\|\nabla u\|_{L^{q_{0}-2}}^{\frac{\beta q_{0}}{\beta q_{0}}}+\|\tau\|_{H^{2+\beta}}\right)\left(\left\|\Lambda^{s} u\right\|_{L^{2}}^{2}+\left\|\Lambda^{s} \tau\right\|_{L^{2}}^{2}\right) \\
& \leq \frac{\eta}{8}\left\|\Lambda^{s+\beta} \tau\right\|_{L^{2}}^{2}+C\left(1+\|\nabla u\|_{L^{q_{0}}}^{2}+\|\tau\|_{H^{2+\beta}}\right)\left(\left\|\Lambda^{s} u\right\|_{L^{2}}^{2}+\left\|\Lambda^{s} \tau\right\|_{L^{2}}^{2}\right),
\end{aligned}
$$

where $2<q_{0}<\infty$. For the case $\beta \in(0,1)$, we have by using the divergence-free condition

$$
\begin{aligned}
& N_{2}=\int_{\mathbb{R}^{2}}\left[\Lambda^{s} \partial_{i}, u_{i}\right] \tau_{k l} \Lambda^{s} \tau_{k l} \mathrm{~d} x \\
& \leq C\left\|\left[\Lambda^{s} \partial_{i}, u\right] \tau\right\|_{L^{2}}\left\|\Lambda^{s} \tau\right\|_{L^{2}} \\
& \leq C\left(\|\nabla u\|_{L^{q_{0}}}\left\|\Lambda^{s} \tau\right\|_{L^{\frac{2 q_{0}}{q_{0}-2}}}+\|\tau\|_{L^{p_{0}}}\left\|\Lambda^{s+1} u\right\|_{L^{\frac{2 p_{0}}{p_{0}-2}}}\right)\left\|\Lambda^{s} \tau\right\|_{L^{2}} \\
& \leq C\left(\|\nabla u\|_{L^{q_{0}}}\left\|\Lambda^{s} \tau\right\|_{L^{2}}^{1-\frac{2}{\beta q_{0}}}\left\|\Lambda^{s+\beta} \tau\right\|_{L^{2}}^{\frac{2}{\beta q_{0}}}+\|\tau\|_{L^{p_{0}}}\left\|\Lambda^{s} u\right\|_{L^{2}}^{1-\frac{p_{0}+2}{\alpha p_{0}}}\left\|\Lambda^{s+\alpha} u\right\|_{L^{2}}^{\frac{p_{0}+2}{\alpha p_{0}}}\right)\left\|\Lambda^{s} \tau\right\|_{L^{2}} \\
& \leq \frac{v}{8}\left\|\Lambda^{s+\alpha} u\right\|_{L^{2}}^{2}+\frac{\eta}{8}\left\|\Lambda^{s+\beta} \tau\right\|_{L^{2}}^{2}+C\left(\|\nabla u\|_{L^{q_{0}}}^{\frac{\beta q_{0}}{\beta q_{0}-2}}+\|\tau\|_{L^{p} p^{(\alpha) p_{0}-2}}^{\frac{2 \alpha p_{0}}{(\alpha)}}\right)\left(\left\|\Lambda^{s} u\right\|_{L^{2}}^{2}+\left\|\Lambda^{s} \tau\right\|_{L^{2}}^{2}\right)
\end{aligned}
$$




$$
\begin{aligned}
\leq & \frac{v}{8}\left\|\Lambda^{s+\alpha} u\right\|_{L^{2}}^{2}+\frac{\eta}{8}\left\|\Lambda^{s+\beta} \tau\right\|_{L^{2}}^{2}+C\left(1+\|\nabla u\|_{L^{q_{0}}}^{2}+\|\tau\|_{L^{p_{0}}}^{\frac{2 \alpha p_{0}}{(\alpha-1) p_{0}-2}}\right) \\
& \times\left(\left\|\Lambda^{s} u\right\|_{L^{2}}^{2}+\left\|\Lambda^{s} \tau\right\|_{L^{2}}^{2}\right),
\end{aligned}
$$

where $\frac{2}{p_{0}}<\alpha-1$ and $\frac{2}{\beta}<q_{0}<\infty$. For the case $\beta=1$ or $\alpha=1$, we have

$$
\begin{aligned}
& N_{2}=\int_{\mathbb{R}^{2}}\left[\Lambda^{s} \partial_{i}, u_{i}\right] \tau_{k l} \Lambda^{s} \tau_{k l} \mathrm{~d} x \\
& \leq C\left\|\left[\Lambda^{s} \partial_{i}, u\right] \tau\right\|_{L^{2}}\left\|\Lambda^{s} \tau\right\|_{L^{2}} \\
& \leq C\left(\|\nabla u\|_{L^{q_{0}}}\left\|\Lambda^{s} \tau\right\|_{L^{\frac{2 q_{0}}{q_{0}-2}}}+\|\tau\|_{L^{\infty}}\left\|\Lambda^{s+1} u\right\|_{L^{2}}\right)\left\|\Lambda^{s} \tau\right\|_{L^{2}} \\
& \leq \frac{v}{8}\left\|\Lambda^{s+\alpha} u\right\|_{L^{2}}^{2}+\frac{\eta}{8}\left\|\Lambda^{s+\beta} \tau\right\|_{L^{2}}^{2}+C\left(1+\|\nabla u\|_{L^{q_{0}}}^{2}+\|\tau\|_{L^{\infty}}^{2}\right)\left(\left\|\Lambda^{s} u\right\|_{L^{2}}^{2}+\left\|\Lambda^{s} \tau\right\|_{L^{2}}^{2}\right),
\end{aligned}
$$

The term $N_{3}$ admits the following bound

$$
\begin{aligned}
N_{3} & \leq\left\|\Lambda^{s-\beta}(\tau \nabla u)\right\|_{L^{2}}\left\|\Lambda^{s+\beta} \tau\right\|_{L^{2}} \\
& \leq C\left(\|\tau\|_{L^{p_{0}}}\left\|\Lambda^{s-\beta} \nabla u\right\| \frac{2 p_{0}}{p^{-2}}+\|\nabla u\|_{L^{q_{0}}}\left\|\Lambda^{s-1} \tau\right\|_{L^{\frac{2 q_{0}}{q_{0}-2}}}\right)\left\|\Lambda^{s+\beta} \tau\right\|_{L^{2}} \\
& \leq C\left(\|\tau\|_{L^{p_{0}}}\left\|\Lambda^{s} u\right\|_{L^{2}}^{1-\frac{1-\beta+\frac{2}{p_{0}}}{\alpha}}\left\|\Lambda^{s+\alpha} u\right\|_{L^{2}}^{\frac{1-\beta+\frac{2}{p_{0}}}{\alpha}}+\|\nabla u\|_{L^{q_{0}}}\|\tau\|_{H^{s}}\right)\left\|\Lambda^{s+\beta} \tau\right\|_{L^{2}} \\
& \leq \frac{v}{8}\left\|\Lambda^{s+\alpha} u\right\|_{L^{2}}^{2}+\frac{\eta}{8}\left\|\Lambda^{s+\beta} \tau\right\|_{L^{2}}^{2}+C\left(\|\tau\|_{L^{p_{0}}}^{\frac{2 \alpha p_{0}}{p_{0}}}+\|\nabla u\|_{L^{q_{0}}}^{2}\right)\left(\left\|\Lambda^{s} u\right\|_{L^{2}}^{2}+\left\|\Lambda^{s} \tau\right\|_{L^{2}}^{2}\right),
\end{aligned}
$$

where $\beta-1<\frac{2}{p_{0}}<1$ and $2<q_{0}<\infty$. The last term can be estimated as follows:

$$
N_{4} \leq \frac{v}{8}\left\|\Lambda^{s+\alpha} u\right\|_{L^{2}}^{2}+\frac{\eta}{8}\left\|\Lambda^{s+\beta} \tau\right\|_{L^{2}}^{2}+C\left(\left\|\Lambda^{s} u\right\|_{L^{2}}^{2}+\left\|\Lambda^{s} \tau\right\|_{L^{2}}^{2}\right) .
$$

Putting together the preceding estimates, we obtain

$$
\begin{aligned}
& \frac{\mathrm{d}}{\mathrm{d} t}\left(\left\|\Lambda^{s} u(t)\right\|_{L^{2}}^{2}+\left\|\Lambda^{s} \tau(t)\right\|_{L^{2}}^{2}\right)+\left\|\Lambda^{s+\alpha} u\right\|_{L^{2}}^{2}+\left\|\Lambda^{s+\beta} \tau\right\|_{L^{2}}^{2} \\
& \leq C\left(1+\|\nabla u\|_{L^{q_{0}}}^{2}+\|\tau\|_{L^{p_{0}}}^{\frac{\alpha p_{0}}{\alpha p_{0}-2}}+\chi_{\{\beta \in(0,1)\}}\|\tau\|_{L^{p_{0}}}^{\frac{2 \alpha p_{0}}{(\alpha-1) p_{0}-2}}+\chi_{\{\beta=1\}}\|\tau\|_{L^{\infty}}^{2}\right. \\
& \left.+\chi_{\{\beta \in(1,2)\}}\|\tau\|_{H^{2+\beta}}\right)\left(\left\|\Lambda^{s} u\right\|_{L^{2}}^{2}+\left\|\Lambda^{s} \tau\right\|_{L^{2}}^{2}\right) \\
& \leq C\left(1+\|\nabla u\|_{L^{q_{0}}}^{2}+\|\tau\|_{L^{p_{0}}}^{\frac{\alpha p_{0}}{\alpha p_{0}^{-2}}}+\chi_{\{\beta \in(0,1)\}}\|\tau\|_{L^{p_{0}}}^{\frac{2 \alpha p_{0}}{(\alpha-1) p_{0}-2}}\right. \\
& +\chi_{\{\beta=1\}}\|\nabla \tau\|_{L^{2}}^{2} \ln \left(e+\left\|\Lambda^{s} u\right\|_{L^{2}}^{2}+\left\|\Lambda^{s} \tau\right\|_{L^{2}}^{2}\right) \\
& \left.+\chi_{\{\beta \in(1,2)\}}\|\tau\|_{H^{2+\beta}}\right)\left(\left\|\Lambda^{s} u\right\|_{L^{2}}^{2}+\left\|\Lambda^{s} \tau\right\|_{L^{2}}^{2}\right) \text {, }
\end{aligned}
$$

we have used the following logarithmic-type Sobolev inequality (see [17])

$$
\|\tau\|_{L^{\infty}} \leq C\left(1+\|\nabla \tau\|_{L^{2}} \sqrt{\ln \left(e+\left\|\Lambda^{\varrho} \tau\right\|_{L^{2}}\right)}\right), \quad \varrho>1 .
$$


Consequently, one gets

$$
\begin{aligned}
& \frac{\mathrm{d}}{\mathrm{d} t}\left(\left\|\Lambda^{s} u(t)\right\|_{L^{2}}^{2}+\left\|\Lambda^{s} \tau(t)\right\|_{L^{2}}^{2}\right)+\left\|\Lambda^{s+\alpha} u\right\|_{L^{2}}^{2}+\left\|\Lambda^{s+\beta} \tau\right\|_{L^{2}}^{2} \\
& \leq C H(t) \ln \left(e+\left\|\Lambda^{s} u\right\|_{L^{2}}^{2}+\left\|\Lambda^{s} \tau\right\|_{L^{2}}^{2}\right)\left(\left\|\Lambda^{s} u\right\|_{L^{2}}^{2}+\left\|\Lambda^{s} \tau\right\|_{L^{2}}^{2}\right),
\end{aligned}
$$

where

$$
\begin{aligned}
& H(t):=1+\|\nabla u(t)\|_{L^{q_{0}}}^{2}+\|\tau(t)\|_{L^{p_{0}}}^{\frac{\alpha p_{0}}{\alpha p_{0}-2}}+\chi_{\{\beta \in(0,1)\}}\|\tau(t)\|_{L^{p_{0}}}^{\frac{2 \alpha p_{0}}{(\alpha-1) p_{0}-2}} \\
& +\chi_{\{\beta=1\}}\|\nabla \tau(t)\|_{L^{2}}^{2}+\chi_{\{\beta \in(1,2)\}}\|\tau(t)\|_{H^{2+\beta}} .
\end{aligned}
$$

It follows from (2.1), (2.7) and (2.16) that

$$
\int_{0}^{t} H(\sigma) d \sigma \leq C\left(t, u_{0}, \tau_{0}\right)
$$

Applying the Log-Gronwall inequality to (2.20), we thus have

$$
\left\|\Lambda^{s} u(t)\right\|_{L^{2}}^{2}+\left\|\Lambda^{s} \tau(t)\right\|_{L^{2}}^{2}+\int_{0}^{t}\left(\left\|\Lambda^{s+\alpha} u(\sigma)\right\|_{L^{2}}^{2}+\left\|\Lambda^{s+\beta} \tau(\sigma)\right\|_{L^{2}}^{2}\right) d \sigma \leq C\left(t, u_{0}, \tau_{0}\right) .
$$

This ends the proof of Theorem 1.1.

\section{The proof of Theorem 1.2}

To begin with, according to Lemma 2.1, we have the following result.

Lemma 3.1 Assume that $\left(u_{0}, \tau_{0}\right)$ satisfies the assumptions stated in Theorem 1.2. Let $(u, \tau)$ be the corresponding smooth solution of (1.3). Then, $(u, \tau)$ obeys the global bound

$$
\|u(t)\|_{L^{2}}^{2}+\|\tau(t)\|_{L^{2}}^{2}+\int_{0}^{t}\left\|\Lambda^{2} \tau(s)\right\|_{L^{2}}^{2} d s \leq C\left(u_{0}, \tau_{0}\right) .
$$

Proof of Lemma 3.1 Recalling (2.2), one gets

$$
\frac{1}{2} \frac{\mathrm{d}}{\mathrm{d} t}\left(\gamma\|u(t)\|_{L^{2}}^{2}+\kappa\|\tau(t)\|_{L^{2}}^{2}\right)+\kappa \eta\left\|\Lambda^{2} \tau\right\|_{L^{2}}^{2}+\kappa \zeta\|\tau\|_{L^{2}}^{2}=\kappa \int_{\mathbb{R}^{2}} Q(\nabla u, \tau): \tau \mathrm{d} x .
$$

It is not difficult to check that

$$
\begin{aligned}
\kappa \int_{\mathbb{R}^{2}} Q(\nabla u, \tau): \tau \mathrm{d} x & \leq C \kappa\|u\|_{L^{2}}\|\nabla(\tau \tau)\|_{L^{2}} \\
& \leq C \kappa\|u\|_{L^{2}}\|\tau\|_{L^{6}}\|\nabla \tau\|_{L^{3}} \\
& \leq C \kappa\|u\|_{L^{2}}\left(\|\tau\|_{L^{2}}^{\frac{2}{3}}\left\|\Lambda^{2} \tau\right\|_{L^{2}}^{\frac{1}{3}}\right)\left(\|\tau\|_{L^{2}}^{\frac{1}{3}}\left\|\Lambda^{2} \tau\right\|_{L^{2}}^{\frac{2}{3}}\right) \\
& =C \kappa\|u\|_{L^{2}}\|\tau\|_{L^{2}}\left\|\Lambda^{\beta} \tau\right\|_{L^{2}} \\
& \leq \frac{\kappa \zeta}{2}\|\tau\|_{L^{2}}^{2}+C \frac{\kappa}{\zeta}\|u\|_{L^{2}}^{2}\left\|\Lambda^{2} \tau\right\|_{L^{2}}^{2} \\
& \leq \frac{\kappa \zeta}{2}\|\tau\|_{L^{2}}^{2}+C \frac{\kappa}{\zeta \gamma}\left(\gamma\|u\|_{L^{2}}^{2}+\kappa\|\tau\|_{L^{2}}^{2}\right)\left\|\Lambda^{2} \tau\right\|_{L^{2}}^{2} .
\end{aligned}
$$


In view of (3.2), we thus have

$$
\begin{aligned}
\frac{\mathrm{d}}{\mathrm{d} t}\left(\gamma\|u(t)\|_{L^{2}}^{2}+\kappa\|\tau(t)\|_{L^{2}}^{2}\right) & +\kappa \eta\left\|\Lambda^{2} \tau\right\|_{L^{2}}^{2}+\kappa \zeta\|\tau\|_{L^{2}}^{2} \\
& \leq C \frac{\kappa}{\zeta \gamma}\left(\gamma\|u\|_{L^{2}}^{2}+\kappa\|\tau\|_{L^{2}}^{2}\right)\left\|\Lambda^{2} \tau\right\|_{L^{2}}^{2} .
\end{aligned}
$$

Noticing condition (1.5) and making use of the argument in proving Lemma 2.1, we immediately obtain (3.1) and thus complete the proof of the lemma.

We are now able to show the following key estimates.

Lemma 3.2 Assume that $\left(u_{0}, \tau_{0}\right)$ satisfies the assumptions stated in Theorem 1.2. Let $(u, \tau)$ be the corresponding smooth solution of $(1.3)$. Then, $(u, \tau)$ obeys the global bounds

$$
\begin{gathered}
\left\|\Lambda^{2} \tau(t)\right\|_{L^{2}}^{2}+\int_{0}^{t}\left\|\Lambda^{4} \tau(s)\right\|_{L^{2}}^{2} d s \leq C\left(t, u_{0}, \tau_{0}\right), \\
\|\omega(t)\|_{L^{\infty}}+\|\tau(t)\|_{L^{\infty}}+\int_{0}^{t}\|\nabla \tau(s)\|_{L^{\infty}} d s \leq C\left(t, u_{0}, \tau_{0}\right) .
\end{gathered}
$$

Proof of Lemma 3.2 Now the vorticity $\omega$ of $(1.3)_{1}$ reads as follows:

$$
\partial_{t} \omega+(u \cdot \nabla) \omega=\kappa \nabla \times \nabla \cdot \tau .
$$

Multiplying (3.6) by $\omega$ yields

$$
\begin{aligned}
\frac{1}{2} \frac{\mathrm{d}}{\mathrm{d} t}\|\omega(t)\|_{L^{2}}^{2} & \leq C\|\nabla \times \nabla \cdot \tau\|_{L^{2}}\|\omega(t)\|_{L^{2}} \\
& \leq C\left\|\Lambda^{2} \tau\right\|_{L^{2}}\|\omega(t)\|_{L^{2}},
\end{aligned}
$$

which implies

$$
\frac{\mathrm{d}}{\mathrm{d} t}\|\omega(t)\|_{L^{2}} \leq C\left\|\Lambda^{2} \tau\right\|_{L^{2}}
$$

Keeping in mind (3.1), we deduce

$$
\|\omega(t)\|_{L^{2}} \leq C\left(t, u_{0}, \tau_{0}\right) .
$$

Thanks to (2.15), we have

$$
\frac{1}{2} \frac{\mathrm{d}}{\mathrm{d} t}\left\|\Lambda^{2} \tau(t)\right\|_{L^{2}}^{2}+\eta\left\|\Lambda^{4} \tau\right\|_{L^{2}}^{2}+\zeta\left\|\Lambda^{2} \tau\right\|_{L^{2}}^{2}=J_{1}+J_{2}+J_{3},
$$

where

$$
\begin{aligned}
& J_{1}=-\int_{\mathbb{R}^{2}} \Lambda^{2} Q(\nabla u, \tau): \Lambda^{2} \tau \mathrm{d} x, \quad J_{2}=-\int_{\mathbb{R}^{2}} \Lambda^{2}(u \cdot \nabla \tau): \Lambda^{2} \tau \mathrm{d} x, \\
& J_{3}=\gamma \int_{\mathbb{R}^{2}} \Lambda^{2} \mathcal{D} u: \Lambda^{2} \tau \mathrm{d} x .
\end{aligned}
$$


It is not difficult to see that

$$
\begin{aligned}
J_{1} & \leq C\|Q(\nabla u, \tau)\|_{L^{2}}\left\|\Lambda^{4} \tau\right\|_{L^{2}} \\
& \leq C\|\tau\|_{L^{\infty}}\|\nabla u\|_{L^{2}}\left\|\Lambda^{4} \tau\right\|_{L^{2}} \\
& \leq C\|\tau\|_{H^{2}}\|\omega\|_{L^{2}}\left\|\Lambda^{4} \tau\right\|_{L^{2}} \\
& \leq \frac{\eta}{8}\left\|\Lambda^{4} \tau\right\|_{L^{2}}^{2}+C\|\omega\|_{L^{2}}^{2}\|\tau\|_{H^{2}}^{2}, \\
J_{2} & \leq C\|\nabla \tau u\|_{L^{2}}\left\|\Lambda^{4} \tau\right\|_{L^{2}} \\
& \leq C\|\nabla \tau\|_{L^{\infty}}\|u\|_{L^{2}}\left\|\Lambda^{4} \tau\right\|_{L^{2}} \\
& \leq C\|\tau\|_{L^{2}}^{\frac{1}{2}}\left\|\Lambda^{4} \tau\right\|_{L^{2}}^{\frac{1}{2}}\|u\|_{L^{2}}\left\|\Lambda^{4} \tau\right\|_{L^{2}} \\
& \leq \frac{\eta}{8}\left\|\Lambda^{4} \tau\right\|_{L^{2}}^{2}+C\|u\|_{L^{2}}^{4}\|\tau\|_{L^{2}}^{2}
\end{aligned}
$$

and

$$
J_{3} \leq C\left\|\Lambda^{4} \tau\right\|_{L^{2}}\|\nabla u\|_{L^{2}} \leq \frac{\eta}{8}\left\|\Lambda^{4} \tau\right\|_{L^{2}}^{2}+C\|\omega\|_{L^{2}}^{2} .
$$

Putting together the above estimates into (3.8) yields

$$
\frac{\mathrm{d}}{\mathrm{d} t}\left\|\Lambda^{2} \tau(t)\right\|_{L^{2}}^{2}+\left\|\Lambda^{4} \tau\right\|_{L^{2}}^{2} \leq C\left(\|u\|_{L^{2}}^{4}+\|\omega\|_{L^{2}}^{2}\right)\left(1+\|\tau\|_{H^{2}}^{2}\right) .
$$

By means of (3.1) and (3.7), it follows that

$$
\left\|\Lambda^{2} \tau(t)\right\|_{L^{2}}^{2}+\int_{0}^{t}\left\|\Lambda^{4} \tau(s)\right\|_{L^{2}}^{2} \mathrm{~d} s \leq C\left(t, u_{0}, \tau_{0}\right) .
$$

We deduce from (3.6) that

$$
\frac{\mathrm{d}}{\mathrm{d} t}\|\omega(t)\|_{L^{\infty}} \leq C\left\|\nabla^{2} \tau\right\|_{L^{\infty}} \leq C\left(\left\|\Lambda^{2} \tau\right\|_{L^{2}}+\left\|\Lambda^{4} \tau\right\|_{L^{2}}\right) .
$$

Using (3.9) and integrating (3.10) in time, we get

$$
\|\omega(t)\|_{L^{\infty}} \leq C\left(t, u_{0}, \tau_{0}\right) .
$$

This ends the proof of the lemma.

Finally, the global $H^{s}$-bound can be established as follows.

Proof To begin with, we have

$$
\frac{1}{2} \frac{\mathrm{d}}{\mathrm{d} t}\left(\left\|\Lambda^{s} u(t)\right\|_{L^{2}}^{2}+\left\|\Lambda^{s} \tau(t)\right\|_{L^{2}}^{2}\right)+\eta\left\|\Lambda^{s+2} \tau\right\|_{L^{2}}^{2} \leq N_{1}+N_{2}+N_{3}+N_{4},
$$

where

$$
\begin{aligned}
& N_{1}:=\int_{\mathbb{R}^{2}}\left[\Lambda^{s}, u \cdot \nabla\right] u \cdot \Lambda^{s} u \mathrm{~d} x, \quad N_{2}:=\int_{\mathbb{R}^{2}}\left[\Lambda^{s}, u \cdot \nabla\right] \tau: \Lambda^{s} \tau \mathrm{d} x \\
& N_{3}:=-\int_{\mathbb{R}^{2}} \Lambda^{s} Q(\nabla u, \tau): \Lambda^{s} \tau \mathrm{d} x, \quad N_{4}:=\kappa \int_{\mathbb{R}^{2}} \Lambda^{s} \nabla \cdot \tau \cdot \Lambda^{s} u \mathrm{~d} x+\gamma \int_{\mathbb{R}^{2}} \Lambda^{s} \mathcal{D} u: \Lambda^{s} \tau \mathrm{d} x .
\end{aligned}
$$


Similarly, we may conclude

$$
\begin{aligned}
& N_{1} \leq C\left\|\left[\Lambda^{s}, u \cdot \nabla\right] u\right\|_{L^{2}}\left\|\Lambda^{s} u\right\|_{L^{2}} \leq C\|\nabla u\|_{L^{\infty}\left\|\Lambda^{s} u\right\|_{L^{2}}^{2},} \\
& N_{2} \leq C\left\|\left[\Lambda^{s}, u \cdot \nabla\right] \tau\right\|_{L^{2}}\left\|\Lambda^{s} \tau\right\|_{L^{2}} \\
& \leq C\left(\|\nabla u\|_{L^{\infty}}\left\|\Lambda^{s} \tau\right\|_{L^{2}}+\|\nabla \tau\|_{L^{\infty}}\left\|\Lambda^{s} u\right\|_{L^{2}}\right)\left\|\Lambda^{s} \tau\right\|_{L^{2}}, \\
& N_{3} \leq\left\|\Lambda^{s-1}(\tau \nabla u)\right\|_{L^{2}}\left\|\Lambda^{s+1} \tau\right\|_{L^{2}}
\end{aligned}
$$

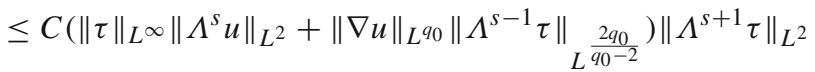

$$
\begin{aligned}
& \leq C\left(\|\tau\|_{L^{\infty}}\left\|\Lambda^{s} u\right\|_{L^{2}}+\|\omega\|_{L^{q_{0}}}\|\tau\|_{H^{s}}\right)\left\|\Lambda^{s} \tau\right\|_{L^{2}}^{\frac{1}{2}}\left\|\Lambda^{s+2} \tau\right\|_{L^{2}}^{\frac{1}{2}} \\
& \leq \frac{\eta}{8}\left\|\Lambda^{s+2} \tau\right\|_{L^{2}}^{2}+C\left(1+\|\tau\|_{L^{\infty}}^{\frac{4}{3}}+\|\omega\|_{L^{q_{0}}}^{\frac{4}{3}}\right)\left(1+\left\|\Lambda^{s} u\right\|_{L^{2}}^{2}+\left\|\Lambda^{s} \tau\right\|_{L^{2}}^{2}\right) \text {, }
\end{aligned}
$$

where $2<q_{0}<\infty$. The last term can be estimated as

$$
N_{4} \leq \frac{\eta}{8}\left\|\Lambda^{s+2} \tau\right\|_{L^{2}}^{2}+C\left(\left\|\Lambda^{s} u\right\|_{L^{2}}^{2}+\left\|\Lambda^{s} \tau\right\|_{L^{2}}^{2}\right) .
$$

Putting together the preceding estimates implies

$$
\begin{aligned}
& \frac{\mathrm{d}}{\mathrm{d} t}\left(\left\|\Lambda^{s} u(t)\right\|_{L^{2}}^{2}+\left\|\Lambda^{s} \tau(t)\right\|_{L^{2}}^{2}\right)+\left\|\Lambda^{s+2} \tau\right\|_{L^{2}}^{2} \\
& \leq C\left(1+\|\nabla u\|_{L^{\infty}}+\|\nabla \tau\|_{L^{\infty}}+\|\tau\|_{L^{\infty}}^{\frac{4}{3}}+\|\omega\|_{L^{q_{0}}}^{\frac{4}{3}}\right)\left(1+\left\|\Lambda^{s} u\right\|_{L^{2}}^{2}+\left\|\Lambda^{s} \tau\right\|_{L^{2}}^{2}\right) \\
& \leq C\left(1+\|\omega\|_{L^{\infty}}+\|\nabla \tau\|_{L^{\infty}}+\|\tau\|_{L^{\infty}}^{\frac{4}{3}}+\|\omega\|_{L^{q_{0}}}^{\frac{4}{3}}\right) \ln \left(e+\left\|\Lambda^{s} u\right\|_{L^{2}}+\left\|\Lambda^{s} \tau\right\|_{L^{2}}\right) \\
& \quad \times\left(e+\left\|\Lambda^{s} u\right\|_{L^{2}}^{2}+\left\|\Lambda^{s} \tau\right\|_{L^{2}}^{2}\right),
\end{aligned}
$$

where we have used the following logarithmic-type Sobolev inequality (see [2])

$$
\|\nabla u\|_{L^{\infty}} \leq C\left(1+\|\omega\|_{L^{\infty}} \ln \left(e+\left\|\Lambda^{s} u\right\|_{L^{2}}\right)\right), \quad s>2 .
$$

Thanks to (3.5), we have that

$$
\int_{0}^{t}\left(1+\|\omega(\sigma)\|_{L^{\infty}}+\|\nabla \tau(\sigma)\|_{L^{\infty}}+\|\tau(\sigma)\|_{L^{\infty}}^{\frac{4}{3}}+\|\omega(\sigma)\|_{L^{q_{0}}}^{\frac{4}{3}}\right) d \sigma \leq C\left(t, u_{0}, \tau_{0}\right) .
$$

The Log-Gronwall inequality implies that

$$
\left\|\Lambda^{s} u(t)\right\|_{L^{2}}^{2}+\left\|\Lambda^{s} \tau(t)\right\|_{L^{2}}^{2}+\int_{0}^{t}\left\|\Lambda^{s+2} \tau(\sigma)\right\|_{L^{2}}^{2} d \sigma \leq C\left(t, u_{0}, \tau_{0}\right) .
$$

This concludes the proof of Theorem 1.2.

\section{The proof of Theorem 1.3}

To begin with, we will establish the following key fact of $\tau$, which plays a key role in proving the main result.

Lemma 4.1 Assume that $\left(u_{0}, \tau_{0}\right)$ satisfies the assumptions stated in Theorem 1.3. If $\beta \in$ $[0,1]$, then $\tau(x, t)$ is a nonnegative definite matrix for any $t \geq 0$, that is, the determinant $\operatorname{Det}(\tau):=\tau_{11} \tau_{22}-\tau_{12}^{2} \geq 0$ and the trace $\operatorname{Tr}(\tau):=\tau_{11}+\tau_{22} \geq 0$. 
Proof of Lemma 4.1 The proof is motivated by [6, Proposition 1]. To this end, we denote

$$
\begin{aligned}
& a(x, t):=\frac{\tau_{11}(x, t)-\tau_{22}(x, t)}{2} ; \\
& b(x, t):=\tau_{12}(x, t)=\tau_{21}(x, t) ; \\
& c(x, t):=\tau_{11}(x, t)+\tau_{22}(x, t) ; \\
& \lambda(x, t):=\frac{\partial_{x_{1}} u_{1}(x, t)-\partial_{x_{2}} u_{2}(x, t)}{2} ; \\
& \mu(x, t):=\frac{\partial_{x_{1}} u_{2}(x, t)+\partial_{x_{2}} u_{1}(x, t)}{2} ; \\
& \omega(x, t):=\partial_{x_{1}} u_{2}(x, t)-\partial_{x_{2}} u_{1}(x, t) .
\end{aligned}
$$

Now keeping in mind $\nabla \cdot u=0$, it follows from the equation $\tau$ of (1.6) that $a, b, c$ satisfy the following equations

$$
\begin{aligned}
& \partial_{t} a+(u \cdot \nabla) a=-b \omega+c \lambda-\zeta a-\eta \Lambda^{2 \beta} a ; \\
& \partial_{t} b+(u \cdot \nabla) b=a \omega+c \mu-\zeta b-\eta \Lambda^{2 \beta} b ; \\
& \partial_{t} c+(u \cdot \nabla) c=4(\lambda a+\mu b)-\zeta c-\eta \Lambda^{2 \beta} c .
\end{aligned}
$$

We define

$$
f(x, t)=c(x, t)-2 \sqrt{a^{2}(x, t)+b^{2}(x, t)} .
$$

By direct computations, one gets

$$
\partial_{t} f+(u \cdot \nabla) f=-2\left(\frac{\zeta}{2}+\frac{\lambda a+\mu b}{\sqrt{a^{2}+b^{2}}}\right) f-\eta \Lambda^{2 \beta} f+2 \eta F(a, b),
$$

where

$$
F(a, b)=\frac{a \Lambda^{2 \beta} a+b \Lambda^{2 \beta} b}{\sqrt{a^{2}+b^{2}}}-\Lambda^{2 \beta} \sqrt{a^{2}+b^{2}} .
$$

For the case $\beta=1$, it gives by (37) of [6] that

$$
F(a, b)=\left(a^{2}+b^{2}\right)^{-\frac{3}{2}} \sum_{j=1}^{2}\left(a \partial_{x_{j}} b-b \partial_{x_{j}} a\right)^{2} \geq 0 .
$$

For the case $\beta=0$, it is obvious that $F(a, b)$ vanishes. For the case $\beta \in(0,1)$, we recall the definition

$$
\Lambda^{2 \beta} g(x)=C_{\beta} \text { P.V. } \int_{\mathbb{R}^{2}} \frac{g(x)-g(y)}{|x-y|^{2+2 \beta}} \mathrm{d} y .
$$

Then, it gives

$$
\begin{aligned}
F(a, b) & =\frac{a \Lambda^{2 \beta} a+b \Lambda^{2 \beta} b-\sqrt{a^{2}+b^{2}} \Lambda^{2 \beta} \sqrt{a^{2}+b^{2}}}{\sqrt{a^{2}+b^{2}}} \\
& =\frac{C_{\beta}}{\sqrt{a^{2}+b^{2}}} \text { P.V. } \int_{\mathbb{R}^{2}} \frac{\sqrt{a^{2}(x)+b^{2}(x)} \sqrt{a^{2}(y)+b^{2}(y)}-a(x) a(y)-b(x) b(y)}{|x-y|^{2+2 \beta}} \mathrm{d} y \\
& \geq 0,
\end{aligned}
$$

where in the last line we have used

$$
\sqrt{a^{2}(x)+b^{2}(x)} \sqrt{a^{2}(y)+b^{2}(y)} \geq a(x) a(y)+b(x) b(y)
$$


by setting $=(a(x), b(x)), \mathbf{f i}=(a(y), b(y))$ and using the simple fact $\cdot \mathbf{f i} \leq \||\mathbf{f i}|$. We thus deduce from (4.1) that

$$
\partial_{t} f+(u \cdot \nabla) f \geq-2\left(\frac{\zeta}{2}+\frac{\lambda a+\mu b}{\sqrt{a^{2}+b^{2}}}\right) f-\eta \Lambda^{2 \beta} f .
$$

Notice that $\tau_{0}$ is a nonnegative symmetric matrix, it yields

$$
\operatorname{Det}(\tau(0)):=\tau_{11}(0) \tau_{22}(0)-\tau_{12}^{2}(0) \geq 0, \quad \tau_{11}(0)+\tau_{22}(0) \geq 0,
$$

which gives

$$
f(0)=2 \sqrt{a^{2}(0)+b^{2}(0)+\operatorname{Det}(\tau(0))}-2 \sqrt{a^{2}(0)+b^{2}(0)} \geq 0 .
$$

Applying the maximum principle to (4.2), it implies that $f(x, t) \geq 0$, namely,

$$
\operatorname{Tr}(\tau) \equiv c(x, t) \geq 2 \sqrt{a^{2}(x, t)+b^{2}(x, t)},
$$

which leads to

$$
\operatorname{Det}(\tau)=\tau_{11} \tau_{22}-\tau_{12}^{2}=\frac{c^{2}-4\left(a^{2}+b^{2}\right)}{4} \geq 0 .
$$

We therefore complete the proof of Lemma 4.1.

With the help of Lemma 4.1, we will prove the following global $L^{2}$-estimate for $(u, \tau)$.

Lemma 4.2 Assume that $\left(u_{0}, \tau_{0}\right)$ satisfies the assumptions stated in Theorem 1.3. Let $(u, \tau)$ be the corresponding smooth solution of (1.6). If $\beta \in(0,1]$, then $(u, \tau)$ obeys the global bounds

$$
\begin{aligned}
& \|u(t)\|_{L^{2}}^{2}+\|\tau(t)\|_{L^{1}}+\int_{0}^{t}\left\|\Lambda^{\alpha} u(s)\right\|_{L^{2}}^{2} d s \leq C\left(t, u_{0}, \tau_{0}\right), \\
& \|\tau(t)\|_{L^{2}}^{2}+\int_{0}^{t}\left\|\Lambda^{\beta} \tau(s)\right\|_{L^{2}}^{2} d s \leq C\left(t, u_{0}, \tau_{0}\right) .
\end{aligned}
$$

Proof of Lemma 4.2 Now we take the trace of the equation $\tau$ and integrate it over the whole space to get

$$
\begin{aligned}
\frac{\mathrm{d}}{\mathrm{d} t} \int_{\mathbb{R}^{2}} \operatorname{Tr}(\tau)(x) \mathrm{d} x+\zeta \int_{\mathbb{R}^{2}} \operatorname{Tr}(\tau)(x) \mathrm{d} x & =\int_{\mathbb{R}^{2}}\left(\tau_{i k} \partial_{x_{k}} u_{i}+\partial_{x_{k}} u_{i} \tau_{k i}\right) \mathrm{d} x \\
& =2 \int_{\mathbb{R}^{2}} \tau_{i k} \partial_{x_{k}} u_{i} \mathrm{~d} x,
\end{aligned}
$$

where we have used the fact

$$
\int_{\mathbb{R}^{2}} \Lambda^{2 \beta} \operatorname{Tr}(\tau)(x) \mathrm{d} x=C_{\beta} \text { P.V. } \int_{\mathbb{R}^{2}} \frac{\operatorname{Tr}(\tau)(x)-\operatorname{Tr}(\tau)(y)}{|x-y|^{2+2 \beta}} \mathrm{d} y=0 .
$$

Multiplying (1.6) 1 by $u$, it gives

$$
\begin{aligned}
\frac{1}{2} \frac{\mathrm{d}}{\mathrm{d} t} \int_{\mathbb{R}^{2}}|u|^{2} \mathrm{~d} x+v \int_{\mathbb{R}^{2}}\left|\Lambda^{\alpha} u\right|^{2} \mathrm{~d} x & =\kappa \int_{\mathbb{R}^{2}}(\nabla \cdot \tau) \cdot u \mathrm{~d} x \\
& =\kappa \int_{\mathbb{R}^{2}} \partial_{x_{k}} \tau_{k i} u_{i} \mathrm{~d} x \\
& =-\kappa \int_{\mathbb{R}^{2}} \tau_{k i} \partial_{x_{k}} u_{i} \mathrm{~d} x \\
& =-\kappa \int_{\mathbb{R}^{2}} \tau_{i k} \partial_{x_{k}} u_{i} \mathrm{~d} x
\end{aligned}
$$


We get by performing $(4.5) \times \kappa+(4.6) \times 2$ that

$$
\frac{\mathrm{d}}{\mathrm{d} t} \int_{\mathbb{R}^{2}}\left(\kappa \operatorname{Tr}(\tau)+|u|^{2}\right) \mathrm{d} x+\int_{\mathbb{R}^{2}}\left(\kappa \zeta \operatorname{Tr}(\tau)+2 \nu\left|\Lambda^{\alpha} u\right|^{2}\right) \mathrm{d} x=0 .
$$

By $c(x, t) \geq 2 \sqrt{a^{2}(x, t)+b^{2}(x, t)}$ of Lemma 4.1, we know that

$$
\int_{\mathbb{R}^{2}} \operatorname{Tr}(\tau) \mathrm{d} x \approx\|\tau(t)\|_{L^{1}}
$$

Therefore, we deduce from (4.7) that

$$
\frac{\mathrm{d}}{\mathrm{d} t}\left(\|u(t)\|_{L^{2}}^{2}+\|\tau(t)\|_{L^{1}}\right)+\left\|\Lambda^{\alpha} u\right\|_{L^{2}}^{2} \leq 0 .
$$

We finally get the desired estimate (4.3) by integrating the above inequality over the time variable. Here, it should be noted that in this section we only consider $\beta \in(0,1]$, as the case $\beta=0$ or $\alpha=2$ will be investigated in Theorem 1.5. Taking the $L^{2}$-inner product of the equation $\tau$ yields

$$
\begin{aligned}
\frac{1}{2} \frac{\mathrm{d}}{\mathrm{d} t}\|\tau(t)\|_{L^{2}}^{2}+\eta\left\|\Lambda^{\beta} \tau\right\|_{L^{2}}^{2}+\zeta\|\tau\|_{L^{2}}^{2} & \leq C\left|\int_{\mathbb{R}^{2}} \tau \tau \nabla u \mathrm{~d} x\right| \\
& \leq C\|\nabla u\|_{L^{\frac{2}{2-\alpha}}}\|\tau \tau\|_{L^{\frac{2}{\alpha}}} \\
& \leq C\left\|\Lambda^{\alpha} u\right\|_{L^{2}}\|\tau\|_{L^{2}}\left\|\Lambda^{\beta} \tau\right\|_{L^{2}} \\
& \leq \frac{\eta}{2}\left\|\Lambda^{\beta} \tau\right\|_{L^{2}}^{2}+C\left\|\Lambda^{\alpha} u\right\|_{L^{2}}^{2}\|\tau\|_{L^{2}}^{2},
\end{aligned}
$$

where in the third line we have used $\alpha+\beta=2$. This implies

$$
\frac{\mathrm{d}}{\mathrm{d} t}\|\tau(t)\|_{L^{2}}^{2}+\left\|\Lambda^{\beta} \tau\right\|_{L^{2}}^{2} \leq C\left\|\Lambda^{\alpha} u\right\|_{L^{2}}^{2}\|\tau\|_{L^{2}}^{2} .
$$

Using (4.3), it follows from the Gronwall inequality that

$$
\|\tau(t)\|_{L^{2}}^{2}+\int_{0}^{t}\left\|\Lambda^{\beta} \tau(s)\right\|_{L^{2}}^{2} \mathrm{~d} s \leq C\left(t, u_{0}, \tau_{0}\right) .
$$

This ends the proof of Lemma 4.2.

Proof of Theorem 1.3 With (4.3) and (4.4) in hand, the remainder proof of Theorem 1.3 is the same as that of Theorem 1.1. We thus omit the details.

\section{The proof of Theorem 1.5}

According to the proof of Lemma 4.1 and Lemma 4.2, it is not difficult to show

$$
\|u(t)\|_{L^{2}}^{2}+\|\tau(t)\|_{L^{1}}+\int_{0}^{t}\|\mathcal{L} u(s)\|_{L^{2}}^{2} \mathrm{~d} s \leq C\left(t, u_{0}, \tau_{0}\right) .
$$

The following estimates are key ingredients to complete the proof of Theorem 1.5.

Lemma 5.1 Assume that $\left(u_{0}, \tau_{0}\right)$ satisfies the assumptions stated in Theorem 1.5. Let $(u, \tau)$ be the corresponding smooth solution of (1.7). Then $(u, \tau)$ obeys the global bound for any $\delta \in(0,1)$

$$
\left\|\Lambda^{\delta} u(t)\right\|_{L^{2}}^{2}+\|\tau(t)\|_{L^{2}}^{2}+\|\tau(t)\|_{L^{\infty}}^{2}+\int_{0}^{t}\left\|\mathcal{L} \Lambda^{\delta} u(s)\right\|_{L^{2}}^{2} d s \leq C\left(t, u_{0}, \tau_{0}\right) .
$$


In particular, we have

$$
\int_{0}^{t}\|\nabla u(s)\|_{L^{\infty}} d s \leq C\left(t, u_{0}, \tau_{0}\right) .
$$

Proof of Lemma 5.1 First, we multiply (1.7) 2 by $|\tau|^{q-2} \tau$ and integrate it over the whole space to show

$$
\frac{1}{q} \frac{\mathrm{d}}{\mathrm{d} t}\|\tau(t)\|_{L^{q}}^{q}+\zeta\|\tau\|_{L^{q}}^{q} \leq C\|\nabla u\|_{L^{\infty}\|\tau(t)\|_{L^{q}}^{q} .} .
$$

This implies

$$
\frac{\mathrm{d}}{\mathrm{d} t}\|\tau(t)\|_{L^{q}}^{2} \leq C\|\nabla u\|_{L^{\infty}}\|\tau(t)\|_{L^{q}}^{2}
$$

for some constant $C$ independent of $q$. Letting $q \rightarrow \infty$ gives

$$
\frac{\mathrm{d}}{\mathrm{d} t}\|\tau(t)\|_{L^{\infty}}^{2} \leq C\|\nabla u\|_{L^{\infty}}\|\tau(t)\|_{L^{\infty}}^{2} .
$$

Thus, it follows that

$$
\frac{1}{2} \frac{\mathrm{d}}{\mathrm{d} t}\left(\|\tau(t)\|_{L^{2}}^{2}+\|\tau(t)\|_{L^{\infty}}^{2}\right) \leq C\|\nabla u\|_{L^{\infty}}\left(\|\tau\|_{L^{2}}^{2}+\|\tau\|_{L^{\infty}}^{2}\right) .
$$

Multiplying equation $(1.7)_{1}$ by $\Lambda^{2 \delta} u$ with $\delta \in(0,1)$, we have

$$
\frac{1}{2} \frac{\mathrm{d}}{\mathrm{d} t}\left\|\Lambda^{\delta} u(t)\right\|_{L^{2}}^{2}+v\left\|\mathcal{L} \Lambda^{\delta} u\right\|_{L^{2}}^{2}=D_{1}+D_{2},
$$

where

$$
D_{1}:=\kappa \int_{\mathbb{R}^{2}} \nabla \cdot \tau \cdot \Lambda^{2 \delta} u \mathrm{~d} x, \quad D_{2}:=\int_{\mathbb{R}^{2}}\left[\Lambda^{\delta}, u \cdot \nabla\right] u \cdot \Lambda^{\delta} u \mathrm{~d} x .
$$

According to the assumptions on $g$ (more precisely, $g$ grows logarithmically), one may conclude that for any fixed $\rho>0$, there exists $N=N(\rho)$ satisfying

$$
g(r) \leq \widetilde{C} r^{\rho}, \quad \forall r \geq N
$$

with the constant $\widetilde{C}=\widetilde{C}(\rho)$. Therefore, we have for any $0<\sigma<2$

$$
\begin{aligned}
\|\mathcal{L} f\|_{L^{2}}^{2} & =\int_{|\xi|<N(\sigma)} \frac{|\xi|^{4}}{g^{2}(|\xi|)}|\widehat{f}(\xi)|^{2} d \xi+\int_{|\xi| \geq N(\sigma)} \frac{|\xi|^{4}}{g^{2}(|\xi|)}|\widehat{f}(\xi)|^{2} d \xi \\
& \geq \int_{|\xi| \geq N(\sigma)} \frac{|\xi|^{4}}{\left[\widetilde{C}|\xi|^{\sigma}\right]^{2}}|\widehat{f}(\xi)|^{2} d \xi \\
& =\int_{\mathbb{R}^{n}} \frac{|\xi|^{4}}{\left[\widetilde{C}|\xi|^{\sigma}\right]^{2}}|\widehat{f}(\xi)|^{2} d \xi-\int_{|\xi|<N(\sigma)} \frac{|\xi|^{4}}{\left[\widetilde{C}|\xi|^{\sigma}\right]^{2}}|\widehat{f}(\xi)|^{2} d \xi \\
& \geq C_{1}\left\|\Lambda^{2-\sigma} f\right\|_{L^{2}}^{2}-C_{2}\|f\|_{L^{2}}^{2},
\end{aligned}
$$

where $C_{1}$ and $C_{2}$ depend only on $\sigma$. As a direct consequence of (5.6), it yields for any $\delta \in(0,1)$

$$
\begin{aligned}
D_{1} & \leq C\|\tau\|_{L^{2}}\left\|\Lambda^{\delta+1} \Lambda^{\delta} u\right\|_{L^{2}} \\
& \leq C\|\tau\|_{L^{2}}\left(\left\|\Lambda^{\delta} u\right\|_{L^{2}}+\left\|\mathcal{L} \Lambda^{\delta} u\right\|_{L^{2}}\right) \\
& \leq \frac{v}{8}\left\|\mathcal{L} \Lambda^{\delta} u\right\|_{L^{2}}^{2}+C\left(\|\tau\|_{L^{2}}^{2}+\left\|\Lambda^{\delta} u\right\|_{L^{2}}^{2}\right) .
\end{aligned}
$$


It follows from (3.11) that

$$
D_{2} \leq C\|\nabla u\|_{L^{\infty}}\left\|\Lambda^{\delta} u\right\|_{L^{2}}^{2} .
$$

Summing up all the above estimates, it follows from (5.4) and (5.5) that

$$
\begin{aligned}
& \frac{\mathrm{d}}{\mathrm{d} t}\left(\left\|\Lambda^{\delta} u(t)\right\|_{L^{2}}^{2}+\|\tau(t)\|_{L^{2}}^{2}+\|\tau(t)\|_{L^{\infty}}^{2}\right)+\left\|\mathcal{L} \Lambda^{\delta} u\right\|_{L^{2}}^{2} \\
& \quad \leq C\left(1+\|\nabla u\|_{L^{\infty}}\right)\left(\left\|\Lambda^{\delta} u\right\|_{L^{2}}^{2}+\|\tau\|_{L^{2}}^{2}+\|\tau\|_{L^{\infty}}^{2}\right) .
\end{aligned}
$$

By the high-low-frequency technique, we have

$$
\|\nabla u\|_{L^{\infty}} \leq\left\|\Delta_{-1} \nabla u\right\|_{L^{\infty}}+\sum_{l=0}^{N-1}\left\|\Delta_{l} \nabla u\right\|_{L^{\infty}}+\sum_{l=N}^{\infty}\left\|\Delta_{l} \nabla u\right\|_{L^{\infty}},
$$

where $\Delta_{l}(l=-1,0,1, \ldots)$ denote the frequency operator (see Appendix for details). Thanks to Lemma A.2, we conclude

$$
\begin{gathered}
\left\|\Delta_{-1} \nabla u\right\|_{L^{\infty}} \leq C\|u\|_{L^{2}}, \\
\sum_{l=N}^{\infty}\left\|\Delta_{l} \nabla u\right\|_{L^{\infty}} \leq C \sum_{l=N}^{\infty} 2^{2 l}\left\|\Delta_{l} u\right\|_{L^{2}} \\
=C \sum_{l=N}^{\infty} 2^{l\left(2-\delta-r_{1}\right)}\left\|\Delta_{l} \Lambda^{\delta+r_{1}} u\right\|_{L^{2}} \\
\leq C 2^{N\left(2-\delta-r_{1}\right)}\left\|\Lambda^{\delta+r_{1}} u\right\|_{L^{2}} \\
\leq C 2^{N\left(2-\delta-r_{1}\right)}\left(\left\|\Lambda^{\delta} u\right\|_{L^{2}}+\left\|\mathcal{L} \Lambda^{\delta} u\right\|_{L^{2}}\right),
\end{gathered}
$$

where we have used $2>r_{1}>2-\delta$. Lemma A. 2 and the Plancherel's theorem allow us to deduce

$$
\begin{aligned}
\sum_{l=0}^{N-1}\left\|\Delta_{l} \nabla u\right\|_{L^{\infty}} & \leq C \sum_{l=0}^{N-1} 2^{2 l}\left\|\Delta_{l} u\right\|_{L^{2}} \\
& \leq C \sum_{l=0}^{N-1}\left\|\Delta_{l} \Delta u\right\|_{L^{2}} \\
& \leq C \sum_{l=0}^{N-1}\left\|\varphi\left(2^{-l} \xi\right)|\xi|^{2} \widehat{u}(\xi)\right\|_{L^{2}} \\
& \leq C \sum_{l=0}^{N-1}\left\|\varphi\left(2^{-l} \xi\right) g(|\xi|) \frac{|\xi|^{2}}{g(|\xi|)} \widehat{u}(\xi)\right\|_{L^{2}} \\
& \leq C \sum_{l=0}^{N-1} g\left(2^{l}\right)\left\|\frac{|\xi|^{2}}{g(|\xi|)} \widehat{\Delta_{l} u(\xi)}\right\|_{L^{2}} \\
& \leq C\left(\sum_{l=0}^{N-1} g^{2}\left(2^{l}\right)\right)^{\frac{1}{2}}\left(\sum_{l=0}^{N-1}\left\|\frac{|\xi|^{2}}{g(|\xi|)} \widehat{\Delta_{l} u(\xi)}\right\|_{L^{2}}^{2}\right.
\end{aligned}
$$




$$
\begin{aligned}
& \leq C g\left(2^{N}\right)\left(\sum_{l=1}^{N-1} 1\right)^{\frac{1}{2}}\left\|\frac{\Lambda^{2}}{g(\Lambda)} u\right\|_{L^{2}} \\
& \leq C g\left(2^{N}\right) \sqrt{N}\|\mathcal{L} u\|_{L^{2}},
\end{aligned}
$$

where we have used the fact that $g$ is a non-decreasing function. We thus have

$$
\|\nabla u\|_{L^{\infty}} \leq C\|u\|_{L^{2}}+C g\left(2^{N}\right) \sqrt{N}\|\mathcal{L} u\|_{L^{2}}+C 2^{N\left(2-\delta-r_{1}\right)}\left(\left\|\Lambda^{\delta} u\right\|_{L^{2}}+\left\|\mathcal{L} \Lambda^{\delta} u\right\|_{L^{2}}\right) .
$$

This together with (5.7) yields

$$
\frac{\mathrm{d}}{\mathrm{d} t} \Gamma(t)+\phi(t) \leq C\left(1+\|u\|_{L^{2}}+g\left(2^{N}\right) \sqrt{N}\|\mathcal{L} u\|_{L^{2}}+2^{N\left(2-\delta-r_{1}\right)}\left(\Gamma^{\frac{1}{2}}(t)+\phi^{\frac{1}{2}}(t)\right)\right) \Gamma(t),
$$

where

$$
\Gamma(t):=\left\|\Lambda^{\delta} u(t)\right\|_{L^{2}}^{2}+\|\tau(t)\|_{L^{2}}^{2}+\|\tau(t)\|_{L^{\infty}}^{2}, \quad \phi(t):=\left\|\mathcal{L} \Lambda^{\delta} u(t)\right\|_{L^{2}}^{2} .
$$

Taking $N$ as

$$
2^{N} \approx(e+\Gamma(t))^{\frac{1}{\aleph}}, \quad \aleph:=2\left(\delta+r_{1}-2\right)>0,
$$

one has

$$
\begin{aligned}
\frac{\mathrm{d}}{\mathrm{d} t} \Gamma(t)+\phi(t) \leq & C\left(1+\|u\|_{L^{2}}+g\left((e+\Gamma(t))^{\frac{1}{\aleph}}\right) \sqrt{\ln (e+\Gamma(t))}\|\mathcal{L} u\|_{L^{2}}\right) \Gamma(t) \\
& +C \Gamma^{\frac{1}{2}}(t) \phi^{\frac{1}{2}}(t) \\
\leq & \frac{1}{2} \phi(t)+C\left(1+\|u\|_{L^{2}}+g\left((e+\Gamma(t))^{\frac{1}{\aleph}}\right) \sqrt{\ln (e+\Gamma(t))}\|\mathcal{L} u\|_{L^{2}}\right) \Gamma(t) .
\end{aligned}
$$

As a result, it directly gives

$$
\frac{\mathrm{d}}{\mathrm{d} t} \Gamma(t)+\phi(t) \leq C(e+\Gamma(t)) \sqrt{\ln (e+\Gamma(t))} g\left((e+\Gamma(t))^{\frac{1}{\aleph}}\right)\left(1+\|u\|_{L^{2}}+\|\mathcal{L} u\|_{L^{2}}\right) .
$$

Now we deduce from (5.8) that

$$
\int_{e+\Gamma(0)}^{e+\Gamma(t)} \frac{\mathrm{d} \tau}{\tau \sqrt{\ln \tau} g\left(\tau^{\frac{1}{\aleph}}\right)} \leq C \int_{0}^{t}\left(1+\|u(\tau)\|_{L^{2}}+\|\mathcal{L} u(\tau)\|_{L^{2}}\right) \mathrm{d} \tau .
$$

It should be noted the following fact due to (1.8)

$$
\int_{e}^{\infty} \frac{\mathrm{d} \tau}{\tau \sqrt{\ln \tau} g\left(\tau^{\frac{1}{\aleph}}\right)}=\sqrt{\aleph} \int_{e^{\frac{1}{\aleph}}}^{\infty} \frac{\mathrm{d} \tau}{\tau \sqrt{\ln \tau} g(\tau)}=\infty .
$$

Due to (5.1), it leads to

$$
\int_{0}^{t}\left(1+\|u(\tau)\|_{L^{2}}+\|\mathcal{L} u(\tau)\|_{L^{2}}\right) \mathrm{d} \tau \leq C\left(t, u_{0}, \tau_{0}\right)
$$

Considering (5.9), (5.10) and (5.11), we therefore conclude that $\Gamma(t)$ is finite for any given finite $t>0$, namely

$$
\Gamma(t) \leq C\left(t, u_{0}, \tau_{0}\right) .
$$

Coming back to (5.8), it also implies

$$
\int_{0}^{t} \phi(\tau) \mathrm{d} \tau \leq C\left(t, u_{0}, \tau_{0}\right)
$$

This completes the proof of Lemma 5.1. 
Finally, we will end this section by establishing the global $H^{s}$-bound.

Proof As before, we first have

$$
\frac{1}{2} \frac{\mathrm{d}}{\mathrm{d} t}\left(\left\|\Lambda^{s} u(t)\right\|_{L^{2}}^{2}+\left\|\Lambda^{s} \tau(t)\right\|_{L^{2}}^{2}\right)+v\left\|\mathcal{L} \Lambda^{s} u\right\|_{L^{2}}^{2} \leq N_{1}+N_{2}+N_{3}+N_{4},
$$

where

$$
\begin{aligned}
& N_{1}:=\int_{\mathbb{R}^{2}}\left[\Lambda^{s}, u \cdot \nabla\right] u \cdot \Lambda^{s} u \mathrm{~d} x, \quad N_{2}:=\int_{\mathbb{R}^{2}}\left[\Lambda^{s}, u \cdot \nabla\right] \tau: \Lambda^{s} \tau \mathrm{d} x \\
& N_{3}:=\int_{\mathbb{R}^{2}} \Lambda^{s}\left(\tau \nabla u+\nabla u^{\top} \tau\right): \Lambda^{s} \tau \mathrm{d} x, \quad N_{4}:=\kappa \int_{\mathbb{R}^{2}} \Lambda^{s} \nabla \cdot \tau \cdot \Lambda^{s} u \mathrm{~d} x .
\end{aligned}
$$

It follows from (5.6) that for any $\sigma \in(0,2)$

$$
\left\|\Lambda^{s+\sigma} u\right\|_{L^{2}} \leq C\left(\left\|\Lambda^{s} u\right\|_{L^{2}}+\left\|\mathcal{L} \Lambda^{s} u\right\|_{L^{2}}\right) .
$$

Noticing this, we may check that

$$
N_{1} \leq C\|\nabla u\|_{L^{\infty}}\left\|\Lambda^{s} u\right\|_{L^{2}}^{2},
$$

$$
\begin{aligned}
& N_{2}=\int_{\mathbb{R}^{2}}\left[\Lambda^{s} \nabla \cdot, u\right] \tau: \Lambda^{s} \tau \mathrm{d} x \\
& \leq C\left\|\left[\Lambda^{s} \nabla, u\right] \tau\right\|_{L^{2}}\left\|\Lambda^{s} \tau\right\|_{L^{2}} \\
& \leq C\left(\|\nabla u\|_{L^{\infty}}\left\|\Lambda^{s} \tau\right\|_{L^{2}}+\|\tau\|_{L^{\infty}}\left\|\Lambda^{s+1} u\right\|_{L^{2}}\right)\left\|\Lambda^{s} \tau\right\|_{L^{2}} \\
& \leq C\left(\|\nabla u\|_{L^{\infty}}\left\|\Lambda^{s} \tau\right\|_{L^{2}}+\|\tau\|_{L^{\infty}}\left\|\Lambda^{s} u\right\|_{L^{2}}+\|\tau\|_{L^{\infty}}\left\|\mathcal{L} \Lambda^{s} u\right\|_{L^{2}}\right)\left\|\Lambda^{s} \tau\right\|_{L^{2}} \\
& \leq \frac{v}{8}\left\|\mathcal{L} \Lambda^{s} u\right\|_{L^{2}}^{2}+C\left(1+\|\nabla u\|_{L^{\infty}}+\|\tau\|_{L^{\infty}}^{2}\right)\left(1+\left\|\Lambda^{s} u\right\|_{L^{2}}^{2}+\left\|\Lambda^{s} \tau\right\|_{L^{2}}^{2}\right), \\
& N_{3} \leq\left\|\Lambda^{s}(\tau \nabla u)\right\|_{L^{2}}\left\|\Lambda^{s} \tau\right\|_{L^{2}} \\
& \leq C\left(\|\tau\|_{L^{\infty}}\left\|\Lambda^{s+1} u\right\|_{L^{2}}+\|\nabla u\|_{L^{\infty}}\left\|\Lambda^{s} \tau\right\|_{L^{2}}\right)\left\|\Lambda^{s} \tau\right\|_{L^{2}} \\
& \leq \frac{v}{8}\left\|\mathcal{L} \Lambda^{s} u\right\|_{L^{2}}^{2}+C\left(1+\|\nabla u\|_{L^{\infty}}+\|\tau\|_{L^{\infty}}^{2}\right)\left(1+\left\|\Lambda^{s} u\right\|_{L^{2}}^{2}+\left\|\Lambda^{s} \tau\right\|_{L^{2}}^{2}\right)
\end{aligned}
$$

and

$$
N_{4} \leq \frac{v}{8}\left\|\mathcal{L} \Lambda^{s} u\right\|_{L^{2}}^{2}+C\left(\left\|\Lambda^{s} u\right\|_{L^{2}}^{2}+\left\|\Lambda^{s} \tau\right\|_{L^{2}}^{2}\right) .
$$

Consequently, we have by combining all the above estimates

$$
\begin{aligned}
\frac{\mathrm{d}}{\mathrm{d} t}\left(\left\|\Lambda^{s} u(t)\right\|_{L^{2}}^{2}+\left\|\Lambda^{s} \tau(t)\right\|_{L^{2}}^{2}\right)+\left\|\mathcal{L} \Lambda^{s} u\right\|_{L^{2}}^{2} \leq & C\left(1+\|\nabla u\|_{L^{\infty}}+\|\tau\|_{L^{\infty}}^{2}\right) \\
& \times\left(1+\left\|\Lambda^{s} u\right\|_{L^{2}}^{2}+\left\|\Lambda^{s} \tau\right\|_{L^{2}}^{2}\right) .
\end{aligned}
$$

It follows from (5.2) and (5.3) that

$$
\int_{0}^{t}\left(1+\|\nabla u(\sigma)\|_{L^{\infty}}+\|\tau(\sigma)\|_{L^{\infty}}^{2}\right) d \sigma \leq C\left(t, u_{0}, \tau_{0}\right) .
$$

Thanks to the Gronwall inequality, it yields

$$
\left\|\Lambda^{s} u(t)\right\|_{L^{2}}^{2}+\left\|\Lambda^{s} \tau(t)\right\|_{L^{2}}^{2}+\int_{0}^{t}\left\|\mathcal{L} \Lambda^{s} u(\sigma)\right\|_{L^{2}}^{2} d \sigma \leq C\left(t, u_{0}, \tau_{0}\right) .
$$

This ends the proof of Theorem 1.5. 
Acknowledgements Part of this work was carried out during the visit of the author to the Department of Mathematics, University of Pittsburgh. The author would like to appreciate the hospitality of Professor Dehua Wang and Professor Ming Chen. The author also would like to thank the anonymous referee and the corresponding editor for their insightful comments and suggestions. This work was supported by the National Natural Science Foundation of China (No. 11701232) and the Natural Science Foundation of Jiangsu Province (No. BK20170224).

\section{Appendix A: Besov spaces}

This appendix recalls the Littlewood-Paley theory, introduces the Besov spaces and provides Bernstein lemma. We start with the Littlewood-Paley theory. We choose some smooth radial non-increasing function $\chi$ with values in $[0,1]$ such that $\chi \in C_{0}^{\infty}\left(\mathbb{R}^{n}\right)$ is supported in the ball $\mathcal{B}:=\left\{\xi \in \mathbb{R}^{n},|\xi| \leq \frac{4}{3}\right\}$ and with value 1 on $\left\{\xi \in \mathbb{R}^{n},|\xi| \leq \frac{3}{4}\right\}$, then we set $\varphi(\xi)=\chi\left(\frac{\xi}{2}\right)-\chi(\xi)$. One easily verifies that $\varphi \in C_{0}^{\infty}\left(\mathbb{R}^{n}\right)$ is supported in the annulus $\mathcal{C}:=\left\{\xi \in \mathbb{R}^{n}, \frac{3}{4} \leq|\xi| \leq \frac{8}{3}\right\}$ and satisfies

$$
\chi(\xi)+\sum_{j \geq 0} \varphi\left(2^{-j} \xi\right)=1, \quad \forall \xi \in \mathbb{R}^{n} .
$$

Let $h=\mathcal{F}^{-1}(\varphi)$ and $\tilde{h}=\mathcal{F}^{-1}(\chi)$; then, we introduce the dyadic blocks $\Delta_{j}$ of our decomposition by setting

$$
\begin{aligned}
& \Delta_{j} u=0, \quad j \leq-2 ; \quad \Delta_{-1} u=\chi(D) u=\int_{\mathbb{R}^{n}} \tilde{h}(y) u(x-y) \mathrm{d} y ; \\
& \Delta_{j} u=\varphi\left(2^{-j} D\right) u=2^{j n} \int_{\mathbb{R}^{n}} h\left(2^{j} y\right) u(x-y) \mathrm{d} y, \quad \forall j \in \mathbb{N} .
\end{aligned}
$$

We shall also use the following low-frequency cutoff:

$$
S_{j} u=\chi\left(2^{-j} D\right) u=\sum_{-1 \leq k \leq j-1} \Delta_{k} u=2^{j n} \int_{\mathbb{R}^{n}} \tilde{h}\left(2^{j} y\right) u(x-y) \mathrm{d} y, \quad \forall j \in \mathbb{N} .
$$

The nonhomogeneous Besov spaces are defined through the dyadic decomposition.

Definition A.1 Let $s \in \mathbb{R},(p, r) \in[1,+\infty]^{2}$. The nonhomogeneous Besov space $B_{p, r}^{s}$ is defined as a space of $f \in S^{\prime}\left(\mathbb{R}^{n}\right)$ such that

$$
B_{p, r}^{s}=\left\{f \in S^{\prime}\left(\mathbb{R}^{n}\right) ;\|f\|_{B_{p, r}^{s}}<\infty\right\},
$$

where

$$
\|f\|_{B_{p, r}^{s}}=\left\{\begin{array}{l}
\left(\sum_{j \geq-1} 2^{j r s}\left\|\Delta_{j} f\right\|_{L^{p}}^{r}\right)^{\frac{1}{r}}, \quad \forall r<\infty, \\
\sup _{j \geq-1} 2^{j s}\left\|\Delta_{j} f\right\|_{L^{p}}, \quad \forall r=\infty .
\end{array}\right.
$$

We now introduce the Bernstein's inequalities, which are useful tools in dealing with Fourier localized functions, and these inequalities trade integrability for derivatives. The following lemma provides Bernstein-type inequalities for fractional derivatives

Lemma A.2 (see [1]) Assume $1 \leq a \leq b \leq \infty$. If the integer $j \geq-1$, then it holds

$$
\left\|\Lambda^{k} \Delta_{j} f\right\|_{L^{b}} \leq C_{1} 2^{j k+j n\left(\frac{1}{a}-\frac{1}{b}\right)}\left\|\Delta_{j} f\right\|_{L^{a}}, \quad k \geq 0 .
$$


If the integer $j \geq 0$, then we have

$$
C_{2} 2^{j k}\left\|\Delta_{j} f\right\|_{L^{b}} \leq\left\|\Lambda^{k} \Delta_{j} f\right\|_{L^{b}} \leq C_{3} 2^{j k+j n\left(\frac{1}{a}-\frac{1}{b}\right)}\left\|\Delta_{j} f\right\|_{L^{a}}, \quad k \in \mathbb{R},
$$

where $C_{1}, C_{2}$ and $C_{3}$ are constants depending on $k, a$ and $b$ only.

\section{References}

1. Bahouri, H., Chemin, J.-Y., Danchin, R.: Fourier analysis and nonlinear partial differential equations. In: Berger, M., de la Harpe, P., Hirzebruch, F., Hitchin, N.J., Hörmander, L., Kupiainen, A., Lebeau, G., Lin, F.-H., Ngô, B.C., Ratner, M., Serre, D., Sinai, Y.G., Sloane, N.J.A., Vershik, A.M., Waldschmid, M. (eds.) Grundlehren der Mathematischen Wissenschaften, vol. 343. Springer, Heidelberg (2011)

2. Beale, J., Kato, T., Majda, A.: Remarks on the breakdown of smooth solutions for the 3-D Euler equations. Commun. Math. Phys. 94, 61-66 (1984)

3. Bejaoui, O., Majdoub, M.: Global weak solutions for some Oldroyd Models. J. Differ. Equ. 254, 660-685 (2013)

4. Chemin, J.-Y., Masmoudi, N.: About lifespan of regular solutions of equations related to viscoelastic fluids. SIAM J. Math. Anal. 33, 84-112 (2001)

5. Chen, Q., Miao, C.: Global well-posedness of viscoelastic fluids of Oldroyd type in Besov spaces. Nonlinear Anal. 68, 1928-1939 (2008)

6. Constantin, P., Kliegl, M.: Note on global regularity for two-dimensional Oldroyd-B fluids with diffusive stress. Arch. Ration. Mech. Anal. 206, 725-740 (2012)

7. Constantin, P., Sun, W.: Remarks on Oldroyd-B and related complex fluid models. Commun. Math. Sci. 10, 33-73 (2012)

8. Elgindi, T., Rousset, F.: Global regularity for some Oldroyd-B type models. Commun. Pure Appl. Math. 68, 2005-2021 (2015)

9. Fang, D., Hieber, M., Zi, R.: Global existence results for Oldroyd-B fluids in exterior domains: the case of non-small coupling parameters. Math. Ann. 357, 687-709 (2013)

10. Fang, D., Zi, R.: Global solutions to the Oldroyd-B model with a class of large initial data. SIAM J. Math. Anal. 48, 1054-1084 (2016)

11. Fernández-Cara, E., Guillén, F., Ortega, R.: Mathematical modeling and analysis of viscoelastic fluids of the Oldroyd kind. Handb. Numer. Anal. 8, 543-661 (2002)

12. Guillopé, C., Saut, J.-C.: Existence results for the flow of viscoelastic fluids with a differential constitutive law. Nonlinear Anal. 15, 849-869 (1990)

13. Guillopé, C., Saut, J.-C.: Global existence and one-dimensional nonlinear stability of shearingmotions of viscoelastic fluids of Oldroyd type. RAIROMod él. Math. Anal. Num ér. 24, 369-401 (1990)

14. Hieber, M., Naito, Y., Shibata, Y.: Global existence results for Oldroyd-B fluids in exterior domains. J. Differ. Equ. 252, 2617-2629 (2012)

15. Hu, D., Lelievre, T.: New entropy estimates for Oldroyd-B and related models. Commun. Math. Sci. 5, 909-916 (2007)

16. Kato, T., Ponce, G.: Commutator estimates and the Euler and Navier-Stokes equations. Commun. Pure Appl. Math. 41, 891-907 (1988)

17. Kozono, H., Ogawa, T., Taniuchi, Y.: The critical Sobolev inequalities in Besov spaces and regularity criterion to some semi-linear evolution equations. Math. Z. 242, 251-278 (2002)

18. Kupferman, R., Mangoubi, C., Titi, E.S.: A Beale-Kato-Majda breakdown criterion for an Oldroyd-B fluid in the creeping flow regime. Commun. Math. Sci. 6, 235-256 (2008)

19. Lei, Z.: On 2D viscoelasticity with small strain. Arch. Ration. Mech. Anal. 198, 13-37 (2010)

20. Lei, Z., Liu, C., Zhou, Y.: Global existence for a 2D incompressible viscoelastic model with small strain. Commun. Math. Sci. 5, 595-616 (2007)

21. Lei, Z., Masmoudi, N., Zhou, Y.: Remarks on the blowup criteria for Oldroyd models. J. Differ. Equ. 248, 328-341 (2010)

22. Lions, P., Masmoudi, N.: Global solutions for some Oldroyd models of non-Newtonian flows. Chin. Ann. Math. Ser. B 21, 131-146 (2000)

23. Oldroyd, J.G.: Non-Newtonian effects in steady motion of some idealized elastico-viscous liquids. Proc. R. Soc. Lond. Ser. A 245, 278-297 (1958)

24. Wu, J., Xu, X., Ye, Z.: Global regularity for several incompressible fluid models with partial dissipation. J. Math. Fluid Mech. 19, 423-444 (2017) 
25. Ye, Z., Xu, X.: Global regularity for the 2D Oldroyd-B model in the corotational case. Math. Methods Appl. Sci. 39, 3866-3879 (2016)

26. Zi, R., Fang, D., Zhang, T.: Global solution to the incompressible Oldroyd-B model in the critical $L^{p}$ framework: the case of the non-small coupling parameter. Arch. Ration. Mech. Anal. 213, 651-687 (2014) 